Article

\title{
A Mix Inexact-Quadratic Fuzzy Water Resources Management Model of Floodplain (IQT-WMMF) for Regional Sustainable Development of Dahuangbaowa, China
}

\author{
Xueting Zeng, Xiaoliu Yang *, Liyang Yu and Huili Chen \\ Environmental Systems Engineering Program, College of Urban and Environmental Science, \\ Peking University, Beijing 100871, China; \\ E-Mails: xtzeng115@163.com (X.Z.); lyu@mail.millikin.edu (L.Y.); hlchen1989@pku.edu.cn (H.C.) \\ * Author to whom correspondence should be addressed; E-Mail: xlyang@urban.pku.edu.cn; \\ Tel.: +86-10-6275-9327.
}

Academic Editor: Markus Disse

Received: 4 February 2015 / Accepted: 1 June 2015 / Published: 12 June 2015

\begin{abstract}
In this study, a mix inexact-quadratic fuzzy water resources management model of floodplain (IQT-WMMF) has developed, through incorporating techniques of credibility-constrained programming (CP), two-stage programming (TP), interval-parameter programming (IPP) and quadratic programming (QP) within a general framework for limited data availability. The IQT-WMMF can provide an effective linkage between system benefit and the associated economic penalty attributed to the violation of the pre-regulated water target under limited data availabilities expressed probabilistic distributions and interval values; meanwhile, imprecise and no-linear economic data would be resolved. The developed method is applied to a real case of planning water resources in the Dahuangbaowa floodplain, China, with the aim to develop a sustainable water resources management in the study region. A number of scenarios with wet land expansion strategies under various credibility levels are analyzed, implying that different policies can lead to varied water-allocation patterns, system benefits, and system-failure risks. The results discover that water deficits and flood damages have brought negative effects on economic development synchronously, which need to effective plans to reduce losses of shortages and floods for achieving higher system benefits. Tradeoffs between economic benefit and system-failure risk can support generating an increased robustness in risk control for water resources allocation under uncertainties, which is beneficial to adjust the current water-allocation sustainably.
\end{abstract}


Keywords: quadratic programming; inexact two-stage programming; credibility-constrained programming; uncertainty; limited data availability; flooding diversion; water resources management

\section{Introduction}

In recent decades, since the damage caused by flood has been extremely severe, flood-diversion zones are often used for mitigating flooding disasters in many countries [1]. However, various human activities still exist and even expand in such a floodplain (i.e., flood-diversion zones) due to the pressures of increasing populations and developing economies. Particularly in some developing countries, annual human activities (e.g., resident, agriculture and industry) on the floodplain can decrease the retention capacity of water bodies, and varied runoff patterns under changing climatic conditions, which results in losses caused by flood exceeding the benefit of the floodplain [2]. These lead to a variety of adverse impacts on development of economy and sustainability of human life [3,4]. Therefore, a sound strategy for water resources planning of floodplain is desired to address challenges of water shortage, flood events, unreliable water supply, and incremental water demand [5]. However, uncertainties existing in planning processes are often impacted by social, economic, environmental, technical, and political factors, which are caused by not only limited data availabilities, but also imitations of artificial cognitions/statistics. Particularly in water resources management of floodplain (WMF), the non-flood and flood periods corresponding to deficit penalties and flooding losses exist in a WMF system synchronously; thus, the complexities of water resources plans would be intensified. Such uncertainties and their interrelationships can bring about enormous troubles and challenges for water managers to generate desired decision alternatives [6]. Consequently, an effective plan of WMF for tackling uncertainties and their interactions is important for facilitating sustainable development in watershed systems [7].

In WMF, multiple uncertainties can be expressed as various manners (e.g., probabilistic, fuzzy and nonlinear manners) caused by random unpredictable nature process, imitations of human understanding and error in accounting. Therefore, numbers of uncertain optimization methods can be introduced to tackle various manners of uncertainties, which can support water managers facing challenges of violated-risk due to uncertainties and their interactions [8-15]. For example, spatial and temporal variations (e.g., stream flows) and frequencies of peaks are associated with the net system benefit that would be the function of the stochastic factors, which can be expressed as probabilistic distributions due to data limited data availability. Meanwhile, water targets of human activities also comprise uncertainties that are associated with economic implications (e.g., deficient penalties) if the promised targets are violated [16]. Therefore, one type of stochastic programming (SP) named two-stage stochastic programming (TSP) can be advocated to handle uncertainties expressed as probabilistic distributions. TSP is effective to provide an effective linkage between policies and the economic penalties, which has advantages in reflecting complexities of system uncertainties as well as analyzing policy scenarios when the pre-regulated targets are violated [17]. However, it cannot handle fuzziness in water resources system due to the lack of data and limitations of human understanding/accounting. For example, water flow may be related to errors in acquired data, variations in spatial and temporal units, and incompleteness or impreciseness of observed 
information in water resources management [18]. Fuzzy programming (FP) is effective to deal with decision problems under fuzzy goal or constraints caused by imprecision and vagueness, when the quality and quantity of uncertain information is often not satisfactory enough to be presented as probabilistic distribution $[19,20]$. However, since many other uncertain components (e.g., economic data, allocation target, and trading ratio) are often not straightforward enough to be expressed as probability/possibility distributions, interval-parameter programming (IPP) can be introduced to deal with uncertainties due to limited data availabilities existing in the model's left- and/or right-hand sides, where interval numbers are acceptable as its uncertain inputs [6,7,21]. Previously, a number of researchers have incorporated TSP, FP and IPP into a framework for water resources management. For example, Maqsood et al. (2005) developed an interval-fuzzy two-stage stochastic programming method for planning water resources management systems associated with multiple uncertainties [16]. Li et al. (2007) proposed a mixed interval-fuzzy two-stage programming method for flooding diversion planning within a multi-reservoir system [5]. Guo et al. (2010) proposed an fuzzy-chance-constrained two-stage mixed-integer linear programming (IFCTIP) method for flood management under multiple uncertainties, which can facilitate dynamic programming for decisions of capacity-expansion planning under fuzzy-stochastic conditions. However, the developed methods had difficulties in dealing with vague information such as errors in acquired data and impreciseness of observed information [1]. Zhang et al. (2011) proposed an inexact credibility-constrained programming (ICP) method for planning water allocation, which could measure fuzzy uncertainties by credibility measure (i.e., an average of the possibility measure and the necessity measure) to improve abilities on encode the vagueness of information [21,22]. However, for a real-world water resources management problem, nonlinear relationships can exist among many system components where revenue and cost parameters are expressed as functions of water demand and supply. The credibility-constrained programming has difficulties in tackling such problems. Quadratic programming (QP) can reflect nonlinearity in cost/benefit objectives, and has global optimum under a number of system conditions [23-26]. Unfortunately, few studies were focused on the IPP, FP and QP method for sustainable WMF planning within a two-stage context previously.

Therefore, the objective of this study is to develop a mix inexact-quadratic fuzzy water resources management model of floodplain (IQT-WMMF). The developed IQT-WMMF method is an integrated optimization technique for tackling multiple uncertainties expressed as discrete intervals, probability distributions, nonlinearity and fuzzy sets. The method is applied to a real case study of water resources management of Dahuangbaowa floodplain, China. The study region is threatened by water deficit and frequent flood contemporarily, where the vulnerable ecological system and human-induced soil erosion/vegetation degradation may intensify conflicts between ecological preservation and economic development in water resources utilization. The obtained results with various credibility levels will be used for identifying optimized water-allocation alternatives, lessening the losses of water deficits and floods, and achieving socioeconomic and ecological sustainability. The method can provide a scientific support for large-scale regional water-resources management under uncertainties at the watershed level.

\section{Methodology}

In a real-world problem of water resources management, uncertainties may be expressed as random variables, which result in the relevant decisions being made under varying probability levels. Such a 
problem can be formulated as a two-stage stochastic programming (TSP) model [4,5]. However, in a water resources management system, the impacts of marginal utility about uncertain benefit and penalty data (i.e., interval values) may lead to the relevant objective function nonlinearity. Interval quadratic programming (IQP) can handle the nonlinearities in the objective function and reflect uncertainty expressed as interval values [23,24]. Therefore, introducing IQP into TSP model, an inexact two-stage quadratic programming (ITQP) model can be formulated as follows:

$$
\operatorname{Max} f^{ \pm}=\sum_{i=1}^{I_{1}}\left(a_{i}^{ \pm} x_{i}^{ \pm}+b_{i}^{ \pm}\right) x_{i}^{ \pm}-\sum_{i=1}^{I_{2}} \sum_{h=1}^{H} p_{h}\left(c_{i}^{ \pm} y_{i}^{ \pm}+e_{i}^{ \pm}\right) y_{i h}^{ \pm}
$$

subject to

$$
\begin{gathered}
\sum_{i=1}^{I_{1}} A_{i j}^{ \pm} x_{i}^{ \pm} \leq B_{j}^{ \pm}, \quad j=1,2, \ldots, J_{1} \\
\sum_{i=1}^{I_{1}} A_{i t}^{ \pm} x_{i}^{ \pm}+\sum_{i=1}^{I_{2}} A_{i t}^{ \pm} x_{i}^{ \pm} \geq w_{h}^{ \pm}, \quad t=1,2, \ldots, J_{2} ; \quad h=1,2, \ldots, H \\
x_{i}^{ \pm} \geq 0, \quad i=1,2, \ldots, I_{1} \\
\mathrm{y}_{i h}^{ \pm} \geq 0, \quad i=1,2, \ldots, I_{2} ; h=1,2, \ldots, H
\end{gathered}
$$

where $x_{i}^{ \pm}$is vector of first-stage decision variables, which presents water demand in a water resources management problem usually [16]. Since water demand (i.e., first-stage decision variables) is often confirmed by decision makers according to empirical value previously, it would be rectified by random event in actual water resources situations in planning periods, which would obtain first-stage benefits $\sum_{i=1}^{I_{1}}\left(a_{i}^{ \pm} x_{i}^{ \pm}+b_{i}^{ \pm}\right) x_{i}^{ \pm}$[25]. $p_{h}$ is probability of random event, where $p_{t h}$ is probability of occurrence for scenario $h[16] . y_{i h}^{ \pm}$is recourse at the second-stage under the occurrence of event (presents water shortages), which obtain expected value of the second-stage penalties $\sum_{i=1}^{I_{2}} \sum_{h=1}^{H} p_{h}\left(c_{i}^{ \pm} y_{i}^{ \pm}+e_{i}^{ \pm}\right) y_{i h}^{ \pm}$[23]. Since economic data are nonlinear in such a water recourses problem, $a_{i}^{ \pm}, b_{i}^{ \pm}, c_{i}^{ \pm}$and $e_{i}^{ \pm}$are used for tackle relevant objective function nonlinearity. $a_{i}^{ \pm}$and $b_{i}^{ \pm}$have a function relation to first-stage variables (i.e., $\left.\left(a_{i}^{ \pm} x_{i}^{ \pm}+b_{i}^{ \pm}\right)\right)$to use for expressing unit system benefit of water being delivered; $c_{i}^{ \pm}$and $e_{i}^{ \pm}$have a function relation to recourse variables (i.e., $\left.\left(c_{i}^{ \pm} y_{i}^{ \pm}+e_{i}^{ \pm}\right)\right)$to serve the purpose of showing unit penalty (i.e., unit loss) of water not being delivered. Therefore, a recourse action between first- and second-stage variables (i.e., $x_{i}^{ \pm}$and $y_{i h}^{ \pm}$) would tend to higher unit system benefit and lower penalty of water resources, which results in optimal values of $f^{ \pm}, A_{i t}^{ \pm}$and $A_{i t}^{ \pm}$are coefficients of $x_{i}^{ \pm}$and $y_{i h}^{ \pm} ; w_{i h}^{ \pm}$is random variable of constraint $i$, which is associated with probability level $p_{h}$ [5]. In Equations (1a)-(1e), the decision variables are divided into two subsets: those that must be determined before the realizations of random variables are disclosed (i.e., $\left.x_{i}^{ \pm}\right)$, and those (recourse variables) that can be determined after the realized random-variable values are available (i.e., $y_{i h}^{ \pm}$). Since some of these coefficients in Equation ( $\left.2 b\right)$ can be fuzzy numbers, credibility theory would be introduced to tackle fuzzy uncertainties [27,28].

Credibility theory was initialized by Liu and Liu [29] in 2002, which is superior to traditional possibility measure and necessity measure to tackle fuzzy uncertainties due to its property of self-dual [30]. 
Let $B_{j}$ be a fuzzy set of imprecise right-hand sides with possibility distributions, Equation (2b) can be expressed as follows:

$$
\operatorname{Cr}\left\{\sum_{i=1}^{I_{1}} A_{i j}^{ \pm} x_{i}^{ \pm} \leq B_{j}^{ \pm}\right\} \geq \lambda, \quad j=1,2, \ldots, J_{1}
$$

where credibility of satisfying of $\sum_{i=1}^{I_{1}} A_{i j}^{ \pm} x_{i}^{ \pm} \leq B_{j}^{ \pm}$should be greater than or equal to level $\lambda . B_{j}^{ \pm}$is a fuzzy set of imprecise right-hand sides with possibility distributions [27]. Let $B_{j}$ be a fuzzy variable with membership function $\mu$, and let $\mu$ and $r$ be real numbers. The possibility of a fuzzy event, characterized by $B_{j} \leq r$, is defined by $\operatorname{Pos}\left\{B_{j} \leq r\right\}=\sup _{u \leq r} \mu(u)$, while the necessity of a fuzzy event, characterized by $B_{j} \leq r$, is defined by $\operatorname{Nec}\left\{B_{j} \leq r\right\}=1-\sup _{u>r} \mu(u)$ [31]. The credibility measure (Cr) is an average of the possibility measure and the necessity measure [32]:

$$
C r\left\{B_{j} \leq r\right\}=\frac{1}{2}\left(\operatorname{Pos}\left\{B_{j} \leq r\right\}+N e c\left\{B_{j} \leq r\right\}\right)
$$

Let $B_{j}=\left(\underline{B}_{j}, B_{j}, \bar{B}_{j}\right)$ be a triangle fuzzy number and it is adopted in this study due to its computational efficiency. Accordingly, the credibility of the constraint $\sum_{i=1}^{I_{1}} A_{i j}^{ \pm} x_{i}^{ \pm} \leq B_{j}^{ \pm}, j=1,2, \ldots, J_{1}$ could be defined as follows [33,34], and the corresponding credibility measures are as follows:

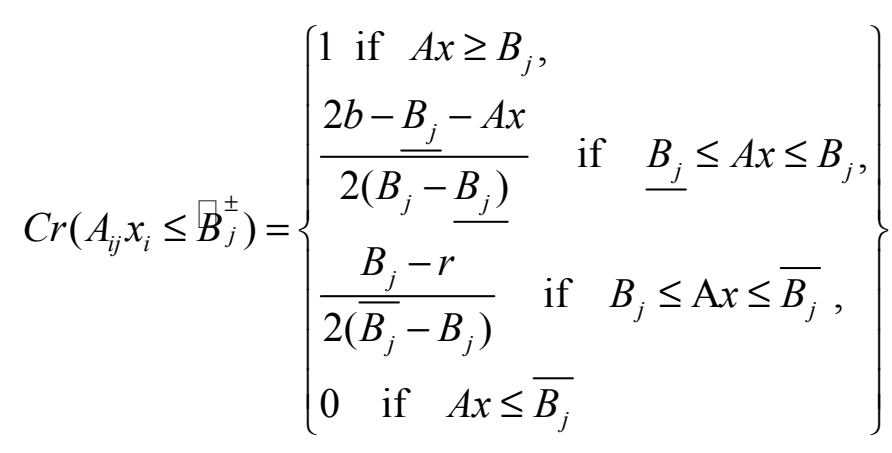

where $\lambda$ is credibility level. In the optimization process for management and planning, it is usually assumed that the credibility level should be no less than 0.5 [35]. Thus, Equation (2a) can be rewritten as:

$$
\operatorname{Cr}\left\{A_{i j} x_{i} \leq B_{j}^{ \pm}\right\}=\frac{2 B_{j}-\underline{B_{j}}-A x}{2\left(B_{j}-\underline{B_{j}}\right)} \geq \lambda
$$

where it can be transformed into a deterministic constraint as follows:

$$
A_{i j} x_{i} \leq B_{j}+(1-2 \lambda)\left(B_{j}-\underline{B_{j}}\right)
$$

Then, an interactive two-step solution algorithm is proposed for solving the inexact-quadratic two-stage programming (IQT) model, which is different from normal interval analysis and best/worst-case analysis $[3,15]$. The IQT model can be transformed into two sets of deterministic submodels, which correspond to the lower and upper bounds of the desired objective function value. The resulting solutions can provide intervals for the objective function and decision variables with different levels of risk in 
violating the constraints. A two-step solution method is proposed for solving the IQT model as shown in Appendix [15,35].

\section{Case Study}

Dahuangbaowa floodplain is located at the junction of Baodi, Wuqing and Ninghe counties of Tianjin province, which is flanked by Hai River (as shown in Figure 1). The Dahuangbaowa floodplain is located in the continental monsoon climate zone, with an average year temperature of $11.5^{\circ} \mathrm{C}$ and average rainfall about $578 \mathrm{~mm}$ per year [36-39]. Precipitation varies largely among different seasons, of which more than $75 \%$ of the total annual precipitation falls from May to September, and less than $20 \%$ of the total falls from November to the following April [40]. Since the floodplain locates at the downstream of Hai River, it is used for mitigating flooding disasters when flood occurs. However, with pressures of high-speed economic development and increased population growth, many human activities can exist even expand in this region. Particular in recent years, agriculture, fishery and livestock are developing at a high speed in this region; while agricultural products processing and manufacturing has been developed due to moderate natural conditions for the growing crops (e.g., wheat, corn, sugar beet, tomato, and fruit), fishing and breeding. Up until 2011, the gross national product (GDP) of floodplain reaches $\$ 0.61$ billion and the population exceeds 46.8 thousand spreading 33 villages [41-44].

Figure 1 shows the division of study regions, which is based Geographic Information System (GIS). In this study, GIS is used to express land use information in study region, where the data of land-use is acquired based on the second national land survey data (2009) [45]. Meanwhile, through the 2765 measured elevation point in the region, the DEM diagram is achieved based on inverse distance weighted interpolation. According to actual terrains and land-use of floodplain, five regions can be divided (i.e., conclude floodplain places and flood buffer areas), where water demands of users (e.g., municipal, agricultural, and ecological users) rely on river's stream-flow. Regions 1 and 2 (denoted as zones 1 and 2) are floodplain places, which are near to the flood discharge valve. They are deemed as diversions for flood discharges during flood time; while they are used for fishery, ecology and wet farm during other time. When the water inflows exceed water-conveyance capacity of river, it may overflow during flooding events. Then water managers would release floods water to diversion regions, which results in submerging zones 1 and 2. Regions 3 to 5 (denoted as zones 3, 4 and 5) (far away from flood discharge valve) are flood buffer areas with aim to buffer flood, which conclude livestock, resident and dry farm. When flood events occur, the released flood water would overwhelm zones 1 and 2 firstly, then it submerge zones 3 to 5 . Since regions 1 and 2 have already relieved the main pressures of flooding, zones 3 to 5 would encounter minor spates (i.e., lower damages) usually, which indicate that important infrastructure and intensive human activities may exist within these regions. Moreover, the depths of the flood diversion regions are different for various kinds of diversion regions. For example, for a lake, the depth of the diversion region can be as high as $50 \mathrm{~m}$, but for a plain place of the diversion region, the depth of the diversion region can only be as low as $2 \mathrm{~m}$. Therefore, the capacities of acceptant flood water can be acquired that zones 1 and 2 are relative low-lying to adopt more flood water, while zones 3 to 5 are high-lying to encounter less released water. 


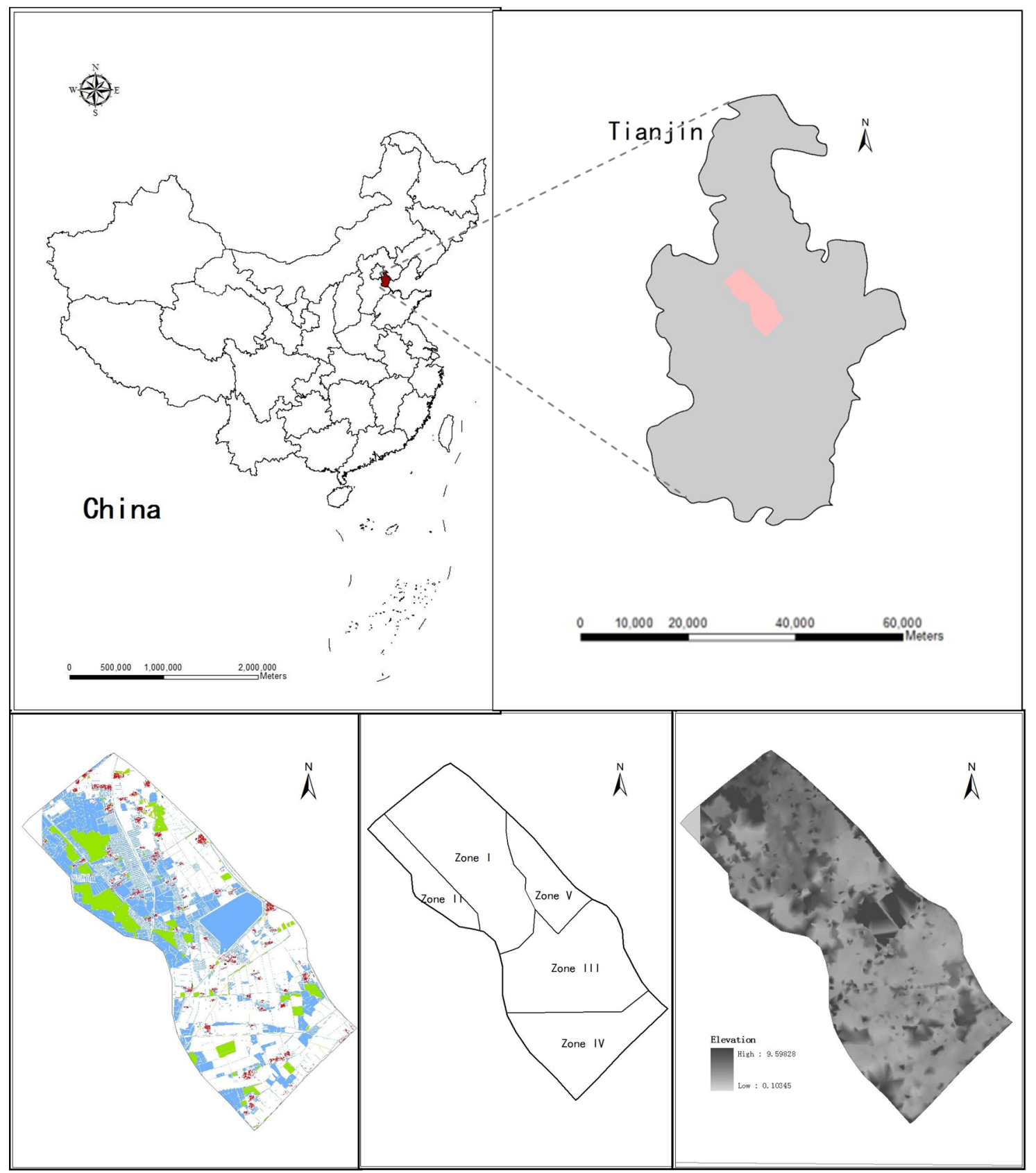

Figure 1. Study area.

Figure 2 presents the framework of IQT-WMMF application of the Dahuangbaowa floodplain. Since the functions of the Dahuangbaowa floodplain conclude flood mitigations and water consumptions, an effective plan to support sustainability in this region can be proposed. In the divided five zones (concluding floodplain and flood buffer), various water users (e.g., ecology, fishery, wet farm, dry farm, fruit farm, livestock and resident) would encounter different risks of flood damages and water deficits. The local authority of floodplain is charge of delivering water to meet demands for the development of regional economy and human life in no-flooding period; meanwhile it is responsible for minimizing the damage of flood when flood occurs. Due to spatial and temporal variations of the relationships between water demand and supply, the desired water-allocation patterns would vary under different strategies of allocation target and outcomes of stream inflow. If water-allocation target pre-regulated by the authority is too high (i.e., higher than the available water), the pre-regulated water could not be successfully 
delivered; shortage would thus be generated. Correspondingly, either the water must be obtained from higher priced alternatives or the demand must be curtailed with reduced production activities, resulting in a decreased net system benefit (i.e., penalty from the shortfalls). On the other hand, if the target as pre-regulated by the authority is too low (i.e., much lower than the available water), more surplus may be generated. When the surplus exceeds the storage capacities of the reservoirs, spill would occur, which might potentially lead to a flooding event. Losses can hardly be avoided when occurs [16]. Meanwhile, since tremendous changes in stream flow in flooding and no-flooding seasons, water shortages and surplus (flood water) would be amplified. Consequently, an optimal strategy for water-allocation target, which is associated with the minimized shortage and surplus, is desired. However, uncertainties exist in many system examples; in a water resources management problem, it is more direct to specify the cost and benefit coefficients within an interval range. Moreover, it is often unreliable to obtain the probability density functions (PDFs) of a random event with probability fit when stream- inflow data are not enough; however, such uncertain data are relatively easy to be expressed as several interval values or fuzzy-interval numbers. These uncertainties and complexities lead to further challenges in identifying optimal water-allocation strategies.

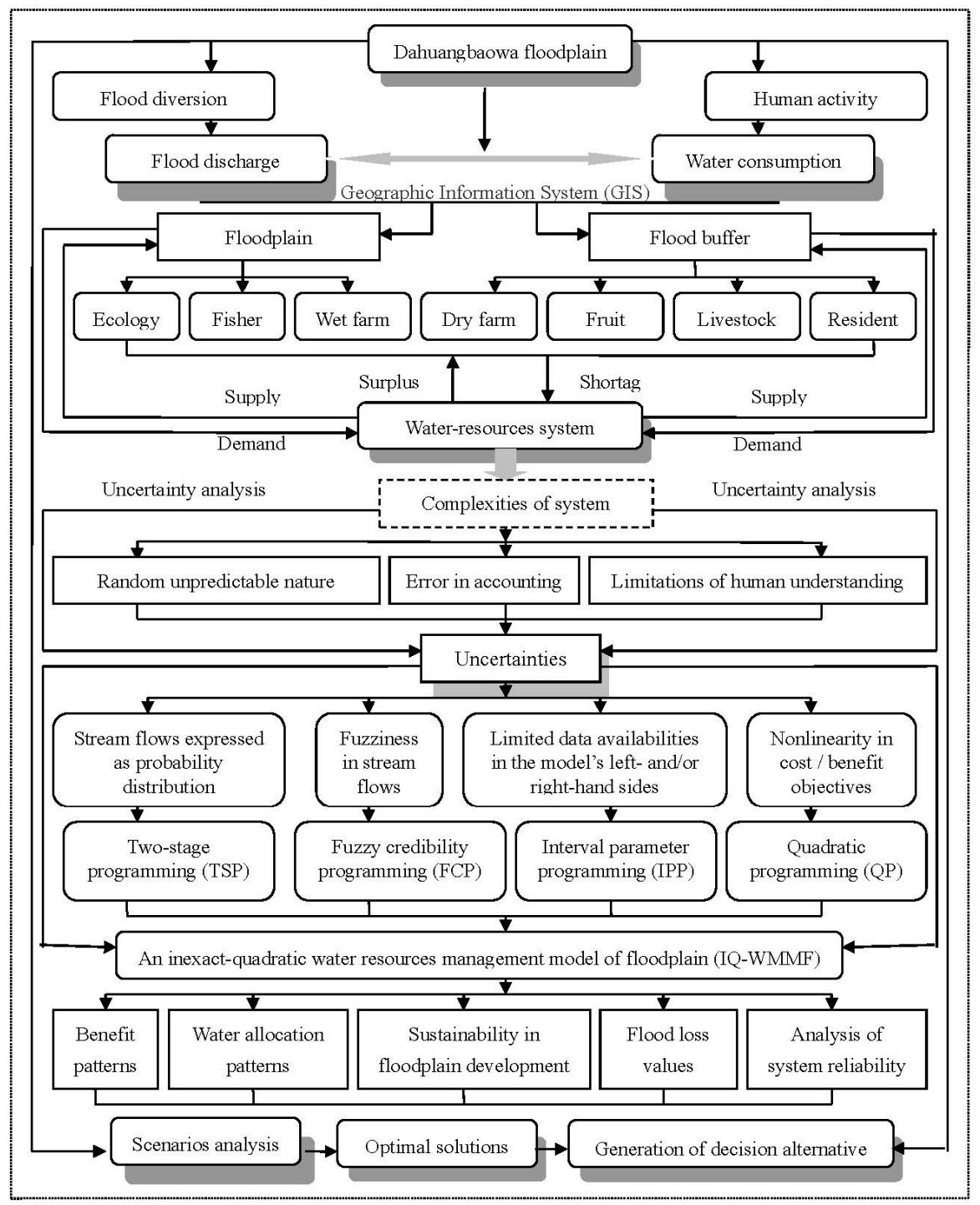

Figure 2. Framework of IQT-WMMF application of Dahuangbaowa floodplain. 
The proposed IQT-WMMF method is considered to be applicable for tackling such a problem. Thus, we have:

$$
\max f^{ \pm}=f_{1}^{ \pm}-f_{2}^{ \pm}-f_{3}^{ \pm}
$$

where $f_{1}^{ \pm}$presents system benefits when water demand targets are delivered (satisfied) $(\$) ; f_{2}^{ \pm}$is losses of water shortage (under probability $p_{j}$ ) in no-flooding season $(\$) ; f_{3}^{ \pm}$is losses of inundation in flooding season (\$). The objective is to maximize the expected net system benefit in no-flooding/flooding season.

$$
\begin{aligned}
f_{1}^{ \pm}= & \sum_{j=1}^{5} X M_{j}^{ \pm}\left(B M_{j}^{a \pm} X M_{j}^{ \pm}+B M_{j}^{b \pm}\right)+\sum_{j=1}^{5} X E_{j}^{ \pm}\left(B E_{j}^{a \pm} X E_{j}^{ \pm}+B E_{j}^{b \pm}\right) I R E_{j}^{ \pm} \\
& +\sum_{j=1}^{5} X W_{j}^{ \pm}\left(B W_{j}^{a \pm} X W_{j}^{ \pm}+B W_{j}^{b \pm}\right) I R W_{j}^{ \pm}+\sum_{j=1}^{5} X D_{j}^{ \pm}\left(B D_{j}^{a \pm} X D_{j}^{ \pm}+B D_{j}^{b \pm}\right) I R D_{j}^{ \pm} \\
& +\sum_{j=1}^{5} X L_{j}^{ \pm}\left(B L_{j}^{a \pm} X L_{j}^{ \pm}+B L_{j}^{b \pm}\right) U R L_{j}^{ \pm}+\sum_{j=1}^{5} X F_{j}^{ \pm}\left(B F_{j}^{a \pm} X F_{j}^{ \pm}+B F_{j}^{b \pm}\right) U P F_{j}^{ \pm} \\
& +\sum_{j=1}^{5} X R_{j}^{ \pm}\left(X R_{j}^{ \pm} B R_{j}^{a \pm}+B R_{j}^{b \pm}\right) U P R_{j}^{ \pm}
\end{aligned}
$$

where $j$ denotes name of region $(j=1,2, \ldots, 5)$. The regions 1 and $2(j=1$ and 2$)$ are floodplain places for flood diversions during flood time; while the regions 3 to $5(j=3$ to 5$)$ are flood buffer areas with aim to buffer flood. $X M_{j}$ is water target for municipal sector in zone $j\left(\mathrm{~m}^{3}\right)$, which is first-stage variable. Similarly, $X E_{j}, X W_{j}, X D_{j}, X F_{j}, X R_{j}$, and $X L_{j}$ are target of irrigation area for ecological, wet farm, dry farm, fishery, fruit farm, livestock sector in zone $j$ (ha). $B M_{j}^{a}$ and $B M_{j}^{b}$ are net benefit parameters to municipal sector in zone $j$ per unit of water being allocated, which have a function relation to first-stage variables (e.g., water targets) to use for tackling nonlinear uncertainties in economic data. Thus, net benefit can be presented as a quadratic programming (QP) expression (i.e., $X M_{j}^{ \pm}\left(B M_{j}^{a \pm} X M_{j}^{ \pm}+B M_{j}^{b \pm}\right)$ ). In the same way, $B E_{j}^{a}, B E_{j}^{b}$, $B W_{j}^{a}, B W_{j}^{b}, B D_{j}^{a}, B D_{j}^{b}, B F_{j}^{a}, B F_{j}^{b}, B R_{j}^{a}, B R_{j}^{b}, B L_{j}^{a}$ and $B L_{j}^{b}$ are net benefit parameters to ecological, wet farm, dry farm, fishery, fruit farm, livestock sector in zone j per unit of water allocated; $I R E_{j}, I R W_{j}, I R D_{j}$, $U R F_{j}, U R R_{j}$, and $U R L_{j}$, are unit water computation of ecological per area $\left(\mathrm{m}^{3} / \mathrm{ha}\right)$.

$$
\begin{aligned}
f_{2}^{ \pm}= & \sum_{h=1}^{7} \sum_{j=1}^{5} p_{h j} C M_{j}^{ \pm}\left(C M_{j}^{ \pm} Y M_{j}^{a \pm}+Y M_{j}^{b \pm}\right)+\sum_{h=1}^{7} \sum_{j=1}^{5} p_{h j} Y E_{j}^{ \pm}\left(C E_{j}^{a \pm} Y E_{j}^{ \pm}+C E_{j}^{b \pm}\right) I R E_{j}^{ \pm} \\
& +\sum_{h=1}^{7} \sum_{j=1}^{5} p_{h j} Y W_{j}^{ \pm}\left(C W_{j}^{a \pm} Y W_{j}^{ \pm}+C W_{j}^{b \pm}\right) I R W_{j}^{ \pm}+\sum_{h=1}^{7} \sum_{j=1}^{5} p_{h j} Y F_{j}^{ \pm}\left(C F_{j}^{a \pm} Y F_{j}^{ \pm}+C F_{j}^{b \pm}\right) U P F_{j}^{ \pm} \\
& +\sum_{h=1}^{7} \sum_{j=1}^{5} p_{h j} Y D_{j}^{ \pm}\left(C D_{j}^{a \pm} Y D_{j}^{ \pm}+C D_{j}^{b \pm}\right) I R D_{j}^{ \pm}+\sum_{h=1}^{7} \sum_{j=1}^{5} p_{h j} Y L_{j}^{ \pm}\left(C L_{j}^{a \pm} Y L_{j}^{ \pm}+C L_{j}^{b \pm}\right) U R L_{j}^{ \pm} \\
& +\sum_{h=1}^{7} \sum_{j=1}^{5} p_{h j} Y R_{j}^{ \pm}\left(Y R_{j}^{ \pm} C R_{j}^{a \pm}+C R_{j}^{b \pm}\right) U P R_{j}^{ \pm}
\end{aligned}
$$

where $h$ denotes probability level of random water availability $(h=1,2, \ldots, 7), h=1$ to 7 represent various water availability levels such as very low (i.e., $V L)$, low (i.e., L), low-medium (i.e., $L M$ ) medium (i.e., $M$ ), medium-high (i.e., $M H)$, high (i.e., $H$ ) and very high (i.e., $V H)$. $p_{h j}$ denotes probability of random water availability $Q_{i j h}^{ \pm}$under level $h(\%)$; Water shortages would occur when water availability levels are relative low, which result in losses in water resources system. $Y M_{j}$ is water shortage for municipal sector 
in zone $j\left(\mathrm{~m}^{3}\right)$, which is second-stage variable; $Y E_{j}, Y W_{j}, Y D_{j}, Y F_{j}, Y R_{j}$ and $Y L_{j}$ are water shortages of irrigation areas for ecology, wet farm, dry farm, fishery, fruit farm, livestock in zone $j$ (ha). $C M_{j}^{a}$ and $C M_{j}^{b}$ are penalties/losses parameters to municipal sector in zone j per unit of water not being allocated. They are used for tackling nonlinear uncertainties in economic data through a function relation to first-stage variables (i.e., $Y M_{j}^{ \pm}\left(C M_{j}^{a \pm} Y M_{j}^{ \pm}+C M_{j}^{b \pm}\right)$ ), which reflecting unit system benefit of water being delivered. Similarly, $C E_{j}^{a}, C E_{j}^{b}, C W_{j}^{a}, C W_{j}^{b}, C D_{j}^{a}, C D_{j}^{b}, C F_{j}^{a}, C F_{j}^{b}, C R_{j}^{a}, C R_{j}^{b}, C L_{j}^{a}$ and $C L_{j}^{b}$ are penalties/losses parameters to ecology, wet farm, dry farm, fishery, fruit farm, livestock in zone $j$ per unit of water allocated.

$$
\begin{aligned}
f_{3}^{ \pm}= & \sum_{h=1}^{3} \sum_{j=1}^{5} p_{h j} D M_{j}^{ \pm} Z M_{j}^{ \pm}+\sum_{h=1}^{3} \sum_{j=1}^{5} p_{h j} D E_{j}^{ \pm} Z E_{j}^{ \pm}+\sum_{h=1}^{3} \sum_{j=1}^{5} p_{h j} D W_{j}^{ \pm} Z W_{j}^{ \pm}+ \\
& \sum_{h=1}^{3} \sum_{j=1}^{5} p_{h j} D F_{j}^{ \pm} Z F_{j}^{ \pm}+\sum_{h=1}^{3} \sum_{j=1}^{5} p_{h j} D L_{j}^{ \pm} Z L_{j}^{ \pm}+\sum_{h=1}^{3} \sum_{j=1}^{5} p_{h j} D D_{j}^{ \pm} Z D_{j}^{ \pm} \\
& +\sum_{h=1}^{3} \sum_{j=1}^{5} p_{h j} D R_{j}^{ \pm} Z R_{j}^{ \pm}
\end{aligned}
$$

when water availability levels are very high even flash floods, damages of floods would result in losses in water resources system. $Z M_{j}$ is water surplus (i.e., flood water) for municipal sector in zone $j\left(\mathrm{~m}^{3}\right)$, which results in losses in flooding seasons. $Z E_{j}, Z W_{j}, Z D_{j}, Z F_{j}, Z R_{j}$ and $Z L_{j}$ are water surplus (i.e., flood water) for ecology, wet farm, dry farm, fishery, fruit farm, livestock in zone $j\left(\mathrm{~m}^{3}\right) . D M_{j}, D E_{j}, D W_{j}, D D_{j}, D F_{j}$, $D R_{j}$, and $D L_{j}$ are losses for municipaityl, ecology, wet farm, dry farm, fishery, fruit farm, livestock in zone $j\left(\$ / \mathrm{m}^{3}\right)$, when flood occurs, which are associated with net system benefits, ratio of water sluice control, direct loss ratio of flood and indirect loss correction coefficient of flood (as shown in Model (4d)).

Constraints include the available water resources, water shortage, flood water, flooding loss, water usage of resident, land use and non-negative as follows:

(1) Constraints of water availability:

$$
Q D_{j h}=Q F_{j h}-E Q_{j h}-H Q_{j h}
$$

Constraint (1) presents the mass balance for water resources system. Where water availability (i.e., $Q D_{j h}$ ) equals water flow (i.e., $Q F_{j h}$ ) minus evaporation/infiltration loss (i.e., $E Q_{j h}^{ \pm}$) and normal water requirement of watercourse (i.e., $\left.H Q_{j h}\right)\left(\mathrm{m}^{3}\right)$. Since data of water flow is limited, $Q F_{j h}$ is a fuzzy number in the Equation (4a), which result in fuzziness in water availability (i.e., $\left.Q D_{j h}\right)$.

(2) Constraints of water shortage:

$$
\begin{aligned}
& \operatorname{Cr}\left\{\sum_{h=1}^{7} \sum_{j=1}^{5}\left(X W_{j}^{ \pm} I R W_{j}^{ \pm}-Y W_{h j}^{ \pm}\right)+\sum_{h=1}^{7} \sum_{j=1}^{5}\left(X F_{j}^{ \pm} U P F_{j}^{ \pm}-Y F_{h j}^{ \pm}\right)+\sum_{h=1}^{7} \sum_{j=1}^{5}\left(X M_{j}^{ \pm}-Y M_{h j}^{ \pm}\right)\right. \\
& +\sum_{h=1}^{7} \sum_{j=1}^{5}\left(X E_{j}^{ \pm} I R E_{j}^{ \pm}-Y E_{h j}^{ \pm}\right)+\sum_{h=1}^{7} \sum_{j=1}^{5}\left(X L_{j}^{ \pm} U R L_{j}^{ \pm}-Y L_{h j}^{ \pm}\right)+\sum_{h=1}^{7} \sum_{j=1}^{5}\left(X D_{j}^{ \pm} I R D_{j}^{ \pm}-Y D_{h j}^{ \pm}\right) \\
& \left.+\sum_{h=1}^{7} \sum_{j=1}^{5}\left(X R_{j}^{ \pm} U P R_{j}^{ \pm}-Y R_{h j}^{ \pm}\right) \leq Q D_{h j}^{ \pm}\right\} \geq \lambda
\end{aligned}
$$


Constraints (2) presents water shortages in no-flooding seasons. Since water demand target is pre-regulated at the beginning of year based on empirical value previously, and pre-regulated water target is not equal to current water availability, a recourse problem would occur to optimize water demand target and system benefit. Equation (4b) reflects that a recourse action has to be undertaken to minimize the reduction of system benefit (i.e., deficiency loss), when the available water resources could not satisfy the water demand.

(3) Constraints of flood water:

$$
\begin{aligned}
& \operatorname{Cr}\left\{Q Q D_{j h}^{ \pm}-\left(\sum_{h=1}^{3} \sum_{j=1}^{5} p_{h j} Z M_{j}^{ \pm}+\sum_{h=1}^{3} \sum_{j=1}^{5} p_{h j} Z E_{j}^{ \pm}+\sum_{h=1}^{3} \sum_{j=1}^{5} p_{h j} Z W_{j}^{ \pm}+\sum_{h=1}^{3} \sum_{j=1}^{5} p_{h j} Z F_{j}^{ \pm}\right.\right. \\
& \left.+\sum_{h=1}^{3} \sum_{j=1}^{5} p_{h j} Z L_{j}^{ \pm}+\sum_{h=1}^{3} \sum_{j=1}^{5} p_{h j} Z D_{j}^{ \pm}+\sum_{h=1}^{3} \sum_{j=1}^{5} p_{h j} Z R_{j}^{ \pm}\right) \geq\left(\sum_{j=1}^{5} X M_{j}^{ \pm}+\sum_{j=1}^{5} X E_{j}^{ \pm} I R E_{j}^{ \pm}\right. \\
& \left.\left.+\sum_{j=1}^{5} X W_{j}^{ \pm} I R W_{j}^{ \pm}+\sum_{j=1}^{5} X D_{j}^{ \pm} I R D_{j}^{ \pm}+\sum_{j=1}^{5} X L_{j}^{ \pm} U R L_{j}^{ \pm}+\sum_{j=1}^{5} X F_{j}^{ \pm} U P F_{j}^{ \pm}+\sum_{j=1}^{5} X R_{j}^{ \pm} U P R_{j}^{ \pm}\right)\right\} \geq \lambda
\end{aligned}
$$

Constraints (3) present flood water in flooding seasons. When water deluges, the water availability has exceed water demand, the surplus water (i.e., flood water) would damage benefits of each water users, which results in extensive losses (concluding indirect and direct losses) in study area.

(4) Constraints of flooding loss:

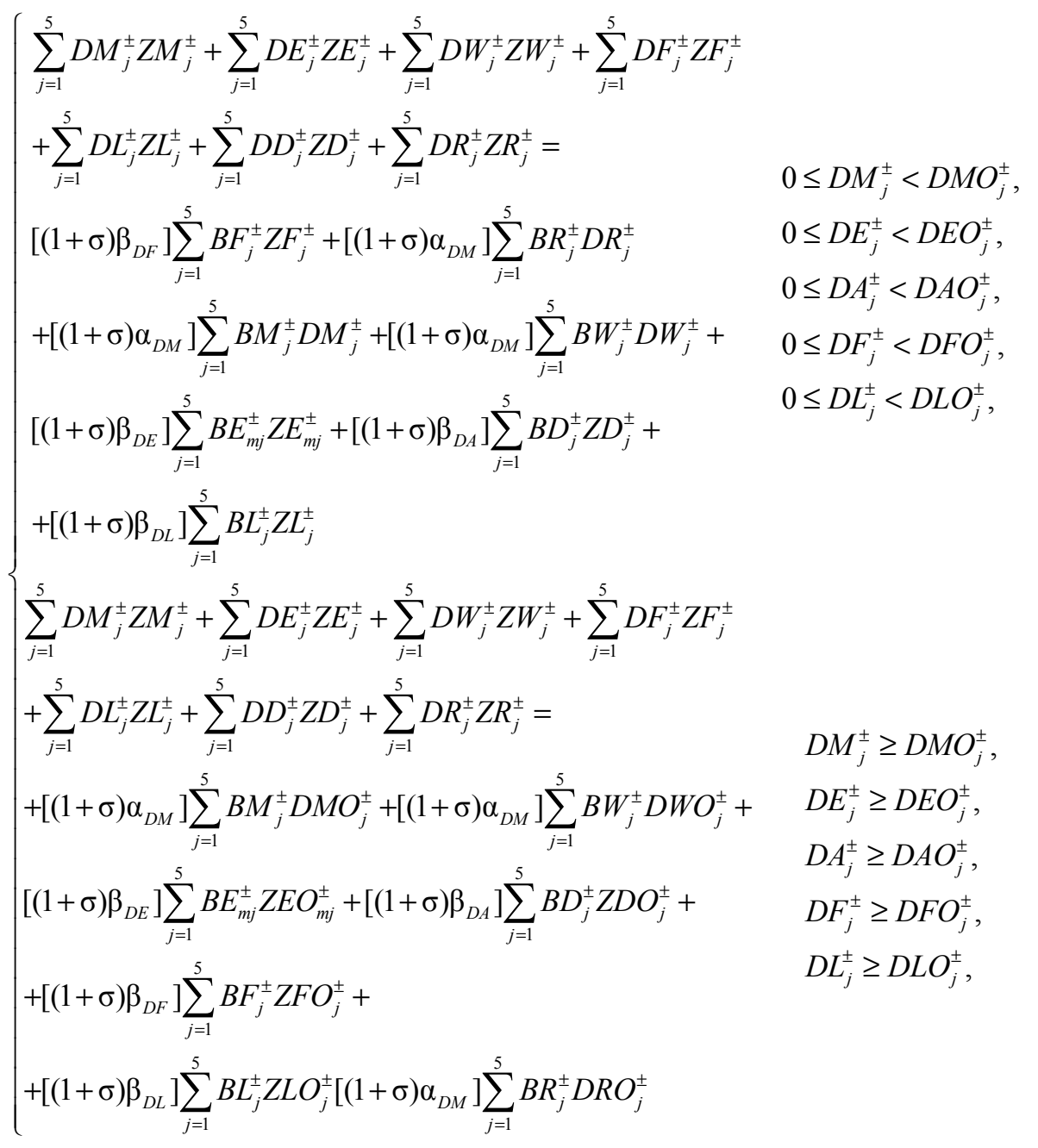


Since extents of damage in water flood are influenced by numbers of factors such as water levels, human land-use of region, sluice control in flood discharge, economic development and indirect/direct loss correction coefficient of flood, the values of flooding losses can be estimated by relative impact factors (e.g., net system benefits, ratio of water sluice control, direct/indirect loss ratio of flood) in study regions. There are two steps in the estimation processes: (a) when flood water is less than maximum damaged water, the values of flooding losses equals to sum of one and $\sigma(i . e$., the indirect loss correction coefficient of flood in district $j$ ) multiplied by $\alpha$ (i.e., the ratio of water sluice control) or $\beta$ (i.e., the direct loss ratio of flood in district $j$ ) based on are net benefits of municipality, ecology, wet farm, dry farm, fishery, fruit farm, livestock in zone $\mathrm{j}$ per unit of water allocated; (b) when flood water is more than maximum damaged water, the values of flooding losses are estimated by $\sigma, \alpha$ and $\beta$ based on are limited unit lost of flood drowning for municipality, ecology, wet farm, dry farm, fishery, fruit farm, livestock in zone $j\left(\$ / \mathrm{m}^{3}\right)$ (i.e., $D M O_{j}, D E O_{j}, D W O_{j}, D D O_{j}, D F O_{j}, D R O_{j}$ and $D L O_{j}$ ). Moreover, since floodplain places (i.e., zone 1 and 2) are deemed as flood diversions during flood time, the ratio of water sluice control would influence damages in these two regions. Under these situations, values of flood losses of fishery, ecology and wet farm (in zone 1 and 2) equal to sum of one and $\sigma$ (i.e., the indirect loss correction coefficient of flood in district $j$ ) multiplied by $\alpha$ (i.e., the ratio of water sluice control) based on net system benefits/limited unit lost of flood drowning for fishery, ecology and wet farm sector in zone $\mathrm{j}\left(\$ / \mathrm{m}^{3}\right)$. In contrast, values of flood losses of livestock, fruit farm, resident and dry farm (in zones 3, 4 and 5) equal to sum of one and $\sigma$ (i.e., the indirect loss correction coefficient of flood in district $j$ ) multiplied by $\beta$ (i.e., the direct loss ratio of flood in district $j$ ) based on net system benefits/limited unit lost of flood drowning for livestock, fruit farm, resident and dry farm.

(5) Constraints of water usage of resident:

$$
\sum_{j=1}^{7} X M_{j}^{ \pm} \geq A X M_{j}^{ \pm}
$$

Constraints (5) is restrictions of water usage of resident, where $A X M_{j}^{ \pm}$is maximum capacity of water usage of resident in study region.

(6) Constraints of land use:

$$
\begin{aligned}
& \sum_{J=1}^{5} X D_{j}^{ \pm} \leq X M D_{j}^{ \pm} \\
& \sum_{J=1}^{5} X W_{j}^{ \pm} \leq X M W_{j}^{ \pm} \\
& \sum_{J=1}^{5} X E_{j}^{ \pm} \leq X M E_{j}^{ \pm} \\
& \sum_{J=1}^{5} X R_{j}^{ \pm} \leq X M R_{j}^{ \pm} \\
& \sum_{J=1}^{5} X F_{j}^{ \pm} \leq X M F_{j}^{ \pm}
\end{aligned}
$$

Constraints (6) are restrictions of land use, which indicates that target of irrigation area for ecological, wet farm, dry farm, fishery, fruit farm, livestock sector (i.e., $X E_{j}, X W_{j}, X D_{j}, X F_{j}$ and $X R_{j}$ ) are less than maximum capacities of land use (i.e., $X M E_{j}, X M W_{j}, X M D_{j}, X M F_{j}$ and $X M R_{j}$ ) (ha). 
(7) Technical constraints:

$$
0 \leq C M_{j}^{ \pm} \leq B M_{j}^{ \pm}, 0 \leq C A_{j}^{ \pm} \leq B A_{j}^{ \pm}, 0 \leq C I_{j}^{ \pm} \leq B I_{j}^{ \pm}, 0 \leq C E_{j}^{ \pm} \leq B E_{j}^{ \pm}
$$

Constraint (7) is technical constraint.

The developed method is applied to a relatively small-scale watershed system where flood water needs to be diverted from a river channel to multiple-diversion regions in a flooding season. In these five regions, water can be delivered to every users to satisfied there pre-regulated water target, shortages and surplus would be occur due to temporal and spatial water inflows. Decision makers desire sound water allocation patterns (no-flooding period) and flood diversion alternatives (flooding period) with both minimized system loss and maximized system safety. Table 1 provides different water flow levels associated with varied probabilities of occurrences, where the system losses (i.e., water deficits and flood damages) are varied with water flows temporally and spatially.

Table 1. Economic data.

\begin{tabular}{|c|c|c|c|c|c|c|c|}
\hline \multirow{2}{*}{ Zone } & \multicolumn{7}{|c|}{ Water Consumer } \\
\hline & Ecology & Fishery & Wet farm & Fruit Farm & Fruit Farm & Livestock & Resident \\
\hline \multicolumn{8}{|c|}{ Net system benefit $\left(\$ 10^{3} / 10^{3} \mathrm{~m}^{3}\right)$} \\
\hline Lower bound & $-48.1 x+208.2$ & $-51.9 x+225.3$ & $-85.3 x+369.3$ & $60.4 x-44.4$ & $186.2 x-180.5$ & $-19.2 x+751.5$ & $8.6 x+804.6$ \\
\hline Upper bound & $-50.7 x+219.1$ & $-54.7 x+237.1$ & $-89.8 x+388.8$ & $63.6 x-46.6$ & $196 x-190$ & $-20.2 x+791.0$ & $9 x+847$ \\
\hline \multicolumn{8}{|c|}{ Loss of water shortage $\left(\$ 10^{3} / 10^{3} \mathrm{~m}^{3}\right)$} \\
\hline Lower bound & $-52.9 x+228.9$ & $-62.4 x+270.4$ & $-102.4 x+443.2$ & $86.5 x-81.1$ & $223.4 x-216.6$ & $-23.0 x+901.8$ & $10.3 x+964.9$ \\
\hline Upper bound & $-55.8 x+241.0$ & $-65.7 x+284.6$ & $-103 x+447.5$ & $76.3 x-55.9$ & $235.2 x-228$ & $-33.8 x+968.2$ & $10.8 x+1016.4$ \\
\hline
\end{tabular}

Table 2 shows water demand target based on water resources bulletin of Tianjin 2000-2013, which is estimated by users' actual water using in recent years while considering the situation of socio-economic development.

Table 2. Water demand target.

\begin{tabular}{cccccccc}
\hline \multirow{2}{*}{ Zone } & \multicolumn{7}{c}{ Water Demand Target $\left(\mathbf{1 0}^{\mathbf{6}} \mathbf{~ m}^{\mathbf{3}}\right)$} \\
\cline { 2 - 7 } & Ecology & Fishery & Wet Farm & Dry Farm & Fruit Farm & Livestock & Resident \\
\hline 1 & {$[418.3,464.8]$} & {$[1410.8,1567.5]$} & {$[100.8,112.0]$} & {$[0,0]$} & {$[0,0]$} & {$[0,0]$} & {$[107.3,119.3]$} \\
2 & {$[184.9,204.6]$} & {$[992.6,1102.9]$} & {$[119.0,132.2]$} & {$[0,0]$} & {$[0,0]$} & {$[0,0]$} & {$[24.3,27.0]$} \\
3 & {$[0,0]$} & {$[0,0]$} & {$[0,0]$} & {$[53.4,59.3]$} & {$[4.5,4.9]$} & {$[45.5,50.5]$} & {$[239.15,265.7]$} \\
4 & {$[0,0]$} & {$[0,0]$} & {$[0,0]$} & {$[57.1,63.4]$} & {$[6.1,6.8]$} & {$[61.4,68.2]$} & {$[299.3,332.5]$} \\
5 & {$[0,0]$} & {$[0,0]$} & {$[0,0]$} & {$[48.9,54.4]$} & {$[5.4,6.1]$} & {$[68.3,75.9]$} & {$[243.0,270.2]$} \\
\hline
\end{tabular}

Table 3 presents economic data concluded net system benefit and loss of water shortage, which are estimated by the statistical yearbook of Tianjin 2000-2013 and the water resources bulletin of Tianjin 2000-2013. When flood water exceed water amount of maximum loss (the losses of flood reach maximum value), the losses of system are estimated by water amount of maximum loss (as shown in Table 3). Therefore, the objective is to minimize the system losses/maximum system benefits through effectively plans coupling water allocation and flood diversion, while incorporating pre-regulated allocation polices within the planning problems. 
Table 3. Probability levels and water flow of right-side hand fuzzy set.

\begin{tabular}{ccccc}
\hline \multirow{2}{*}{ Level } & \multirow{2}{*}{ Probability } & \multicolumn{3}{c}{ Water Flow (106 $\left.\mathbf{~ m}^{\mathbf{3}}\right)$} \\
\cline { 3 - 5 } & & $\underline{Q F_{j h}}$ & $Q F_{j h}$ & $\overline{Q F_{j h}}$ \\
\hline Very low & 0.05 & 4246.5 & 4340.2 & 4470 \\
Low & 0.10 & 4511.9 & 4628.3 & 4749.4 \\
Low-medium & 0.15 & 4777.4 & 4890 & 5028.8 \\
Medium & 0.40 & 5308.2 & 5400.3 & 5587.5 \\
Medium-high & 0.15 & 6369.8 & 6564.1 & 6705.1 \\
High & 0.10 & 11,678 & $11,998.7$ & $12,292.6$ \\
Very high & 0.05 & $21,323.7$ & $21,986.4$ & $22,350.2$ \\
\hline
\end{tabular}

\section{Results and Discussion}

\subsection{Results Analysis}

\subsubsection{Results under Basic Scenario}

In this study, seven stream levels (i.e., very low, low, low-medium, medium, medium-high, high and very high denoted as "VL", "L", "LM", "MH", "H" and "VH") and 3 scenarios of water policies (i.e., scenario 1 to 3 ) corresponding to various water demand and land utilization structure are considered. The solutions obtained under basic scenario (i.e., scenario 1) are presented in Figures 3-6, where multiple uncertainties (i.e., probability distributions, fuzzy sets and intervals) can be facilitated to obtain multiple decision alternatives. Figure 3 presents the optimized water targets of seven competitive water users (i.e., ecology, wet farm, fishery, dry farm, fruit farm, livestock, resident) of five regions under basic scenario, when credibility level is 0.6 . The results illustrate that the lower and upper bounds of optimized targets corresponding to various system benefits and system-failure risks would result in be obtained, which affect water strategies in water allocation patterns. For example, the optimized ecology targets in zone 1 would be $464.8 \times 10^{6} \mathrm{~m}^{3}$, which approaches their upper bounds; this is because it can bring about higher system benefits when their demands are satisfied; thus, the manager have to promise upper-bound quantities to them. Meanwhile, if water cannot be delivered, it generates higher losses, which means higher system-failure risks. In comparison, the optimized wet farm targets in Zone 2 would approach their lower bounds (i.e., $59.3 \times 10^{6} \mathrm{~m}^{3}$ ); this is because the ecological sector is associated with lower benefit levels.
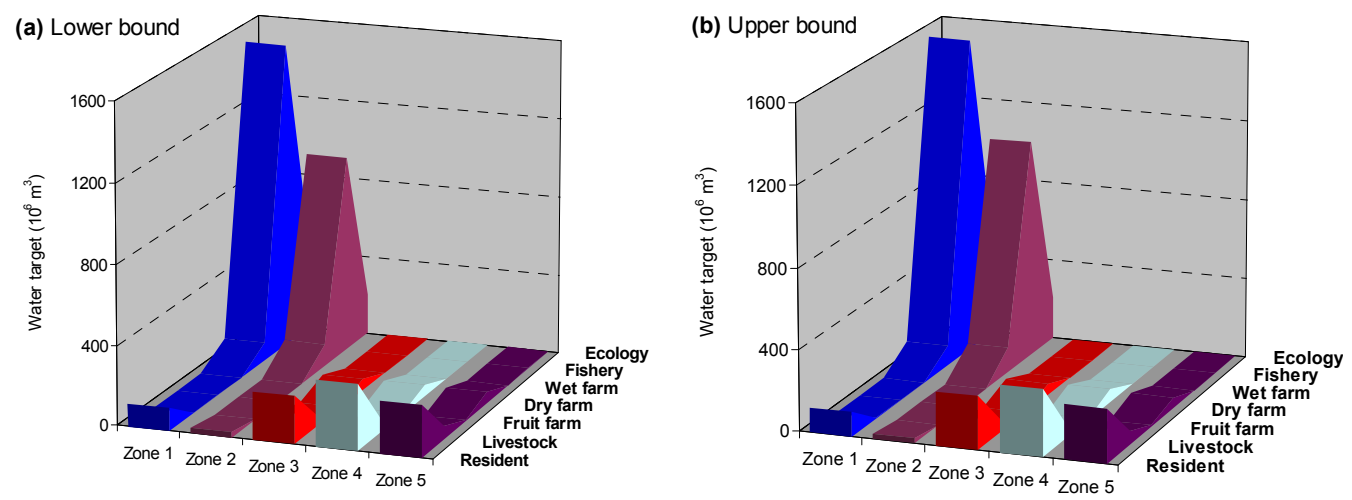

Figure 3. Optimized water targets under scenario $1(\lambda=0.6)$. 
In no flooding reasons are water shortages obtained by inputting the interval numbers of stream flow and the economic data, which indicates that shortage would occur due to limited water availabilities and increased water targets; meanwhile, surplus would occur if the stream inflows are high while the allocation targets pre-regulated are low. Figure 4 presents water shortages and surplus in the study basin under scenario 1. The results indicate that, when the flow is VL in the dry season, the shortage would be tremendous, which would be remitted by increasing of water flow. For example, in Zone 3, water shortages of dry farm would be $[20.8,26.9] \times 10^{6} \mathrm{~m}^{3}$ under VL; while it would be $0 \times 10^{6} \mathrm{~m}^{3}$ under MH. However, when the flow is high in the flood season, the surplus water may be relatively high, which result in damages; and the situations would be enhanced when the flow is very high. For example, in Zone 2, surplus of wet farm would be $0 \times 10^{6} \mathrm{~m}^{3} \times 10^{6} \mathrm{~m}^{3}$ under VL; while it would be [3248.2, 3943.3] $\times 10^{6} \mathrm{~m}^{3}$ under VH. Surplus would occur if the stream inflows are high while the allocation targets pre-regulated are low; under this condition, a water-diversion project would be undertaken to avoid the surplus flow spill over the reservoirs. Due to the functional partitioning of the region, in floodplain (i.e., Zone 1 and 2), a lower target level would result in a higher surplus (and thus a higher flood-diversion loss), particularly when the stream inflow is VL; while the damages of flood would be relived in the flood buffer (i.e., Zone 3 to 5).
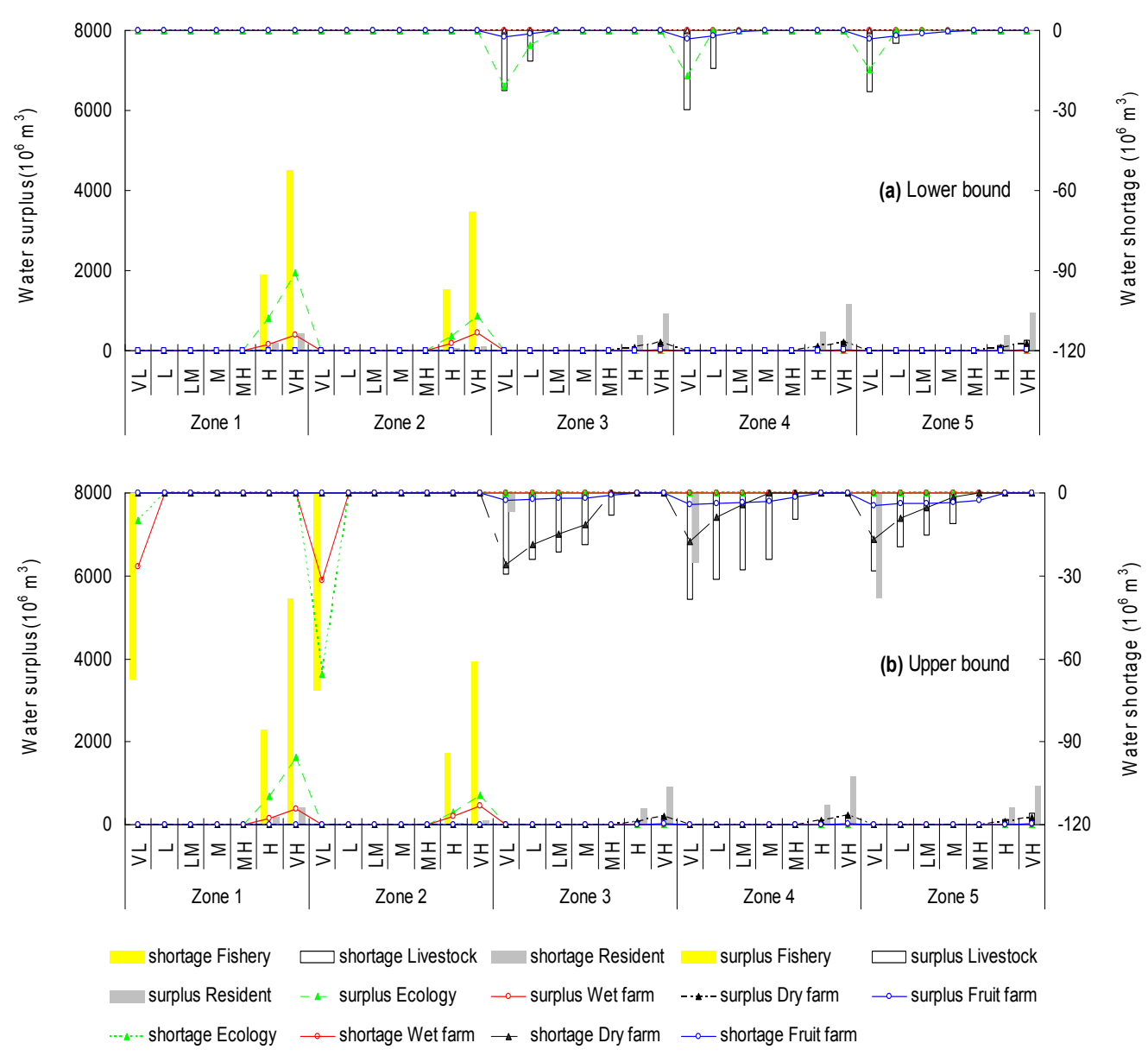

Figure 4. Water shortages and surplus under scenario $1(\lambda=0.6)$.

If a deficit occurs in no flooding period, the actual water allocation would be the difference between the pre-regulated target and the water shortage (i.e., allocation $=$ target - shortage); while the water 
allocation would be exceed water target in flooding season, which lead to damage. Figure 5 shows the optimized water allocations under different water flow seasons $(\lambda=0.6)$. The results indicate that water allocations would vary with water inflow, the lowest water allocation would be achieved when the flow is VL. For example, in Zone 4, water allocation of livestock would be $[27.8,53.1] \times 10^{6} \mathrm{~m}^{3}$ under VL, while it would be $[66.5,68.2] \times 10^{6} \mathrm{~m}^{3}$ under VH. By increasing of water inflows, water allocation would be raised. Due to Zone 1 and 2 are near to outlet of water inflow, and there are many wet lands in these regions, thus the allocations of them would be achieved the optimal targets when the flow is low-medium level. Meanwhile, the water allocations of Zone 3 to 5 would arise to the optimal targets when the flow is at a medium-high level. For example, in Zone 1, water allocation of wet farm would be $[66.7,100.8] \times 10^{6} \mathrm{~m}^{3}$ under VL, while it would be $[95.4,100.8] \times 10^{6} \mathrm{~m}^{3}$ under $\mathrm{L}$ and $\mathrm{VH}$, which indicate it would be the optimal target under L. Meanwhile, in Zone 5, water allocation of fruit farm would be $[1.05,2.46] \times 10^{6} \mathrm{~m}^{3}$ under VL, while it would be $5.44 \times 10^{6} \mathrm{~m}^{3}$ under $\mathrm{H}$ and $\mathrm{VH}$, which indicate it would be the optimal target under $\mathrm{H}$.
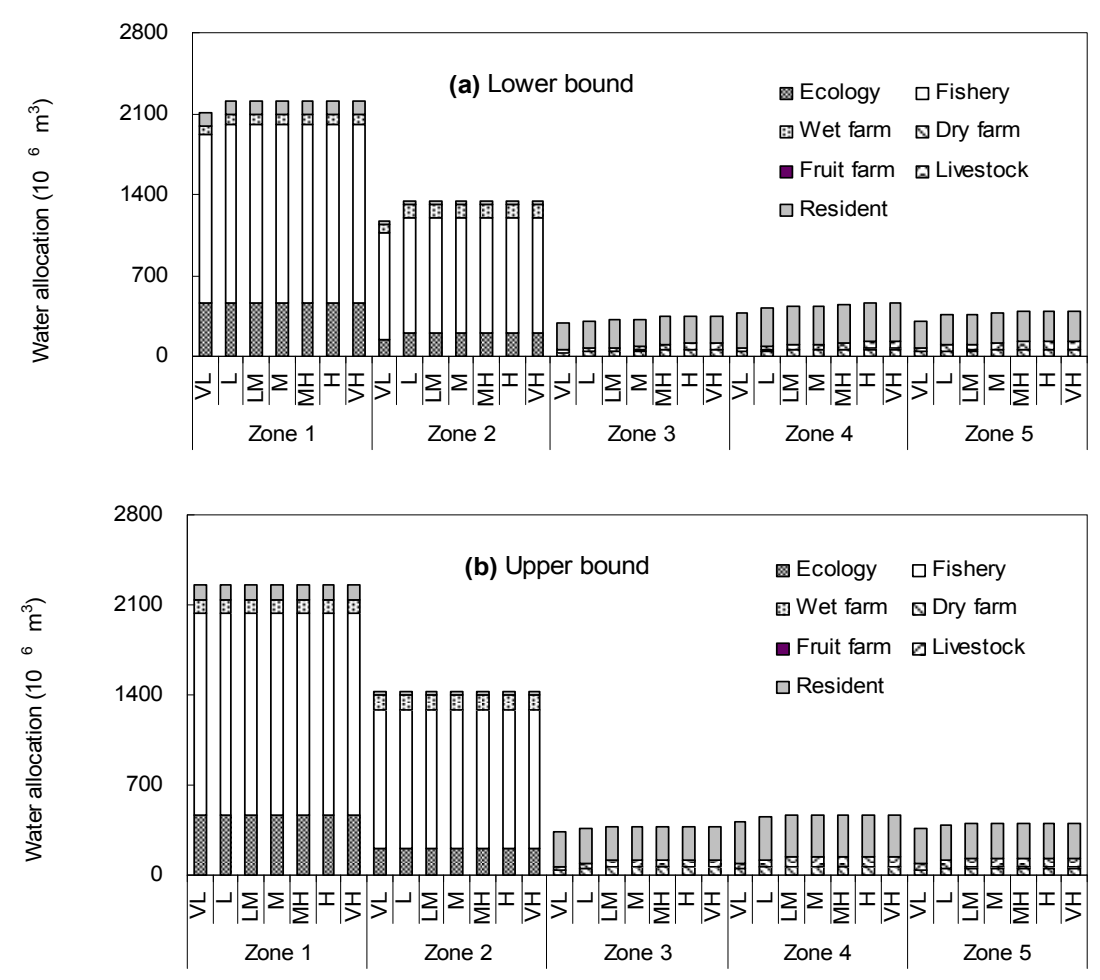

Figure 5. Water allocation under scenario $1(\lambda=0.6)$.

Moreover, several $\lambda$ levels were examined by different combinations of the fuzzy sets, which achieved different water availabilities and varied water-allocations under scenario 1 (as shown in Figure 6). When $\lambda=0.6$, higher water availability corresponded to the lower credibility satisfaction levels would lead to lower water deficiency, which produced higher water allocation. While $\lambda=0.9$, lower water availability corresponded to the higher credibility satisfaction levels would produce the opposite result. Therefore, the amount of water allocation when $\lambda=0.6$ is greater than that when $\lambda=0.9$, implying that a low credibility level could result in a high water-allocation level. For example, water allocations of fishery in Zone 2 would be $[876.4,921.5] \times 10^{6} \mathrm{~m}^{3}$ under VL level when $\lambda=0.6$, while they would be $[787.1,829.3] \times 10^{6} \mathrm{~m}^{3}$ when $\lambda=0.9$. Meanwhile $\lambda=0.9$, lower water resource permit and water availability would correspond 
to the higher credibility satisfaction levels. It replied that different $\lambda$ levels led to different credibility satisfactions and violation risks in water planning system, corresponding to different water availabilities, which lead to different shortages, and allocations.

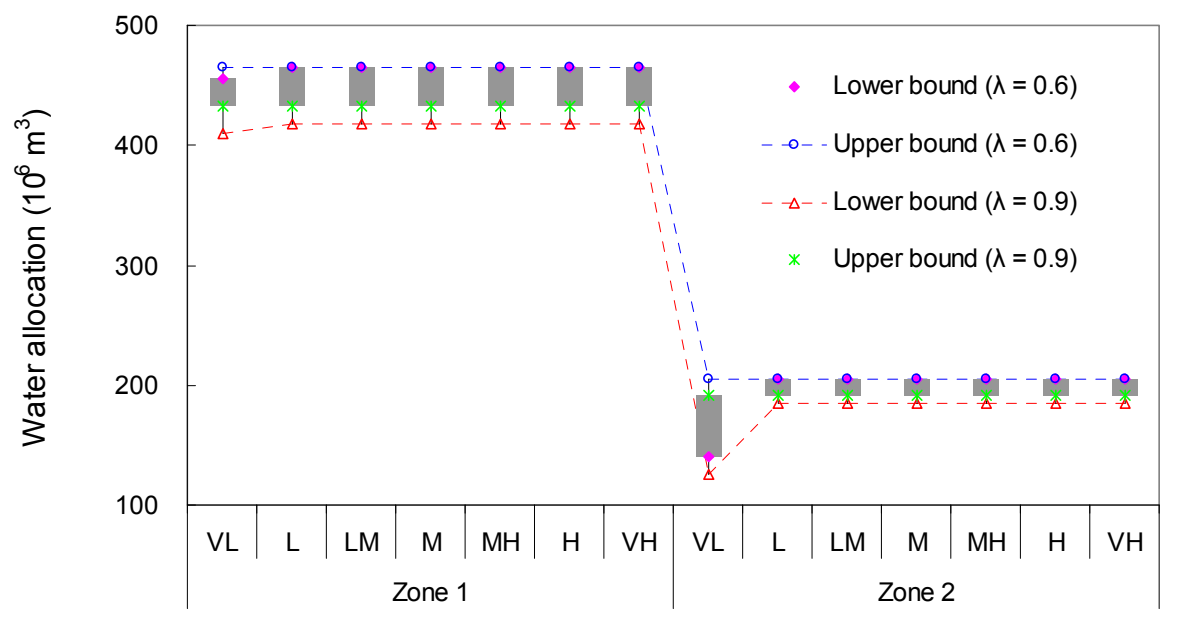

Figure 6. Water allocation of ecology under various $\lambda$-cut levels (scenario 1).

Figure 7 shows the solutions for optimized net system benefits obtained from the IQT-WMMF model, which are the sum of the first-stage benefit from the water allocation and the second-stage random losses of water deficiency. The results demonstrate that different $\lambda$ levels result in varied system benefits, which can reflect expected system benefit preference and risk-averse attitude of decision makers. For example, net system benefits would be achieved $[36.4,46.7] \times 109 \$(\lambda=0.6)$ and $[30.6,39.7] \times 10^{9} \$(\lambda=0.9)$ under scenario 1. It implies that the lower-bound system benefits could result in a lower risk of violating system; reversely a higher benefit would lead to a higher probability of violating system. Consequently, there is a tradeoff between the net system benefits and violation risk.

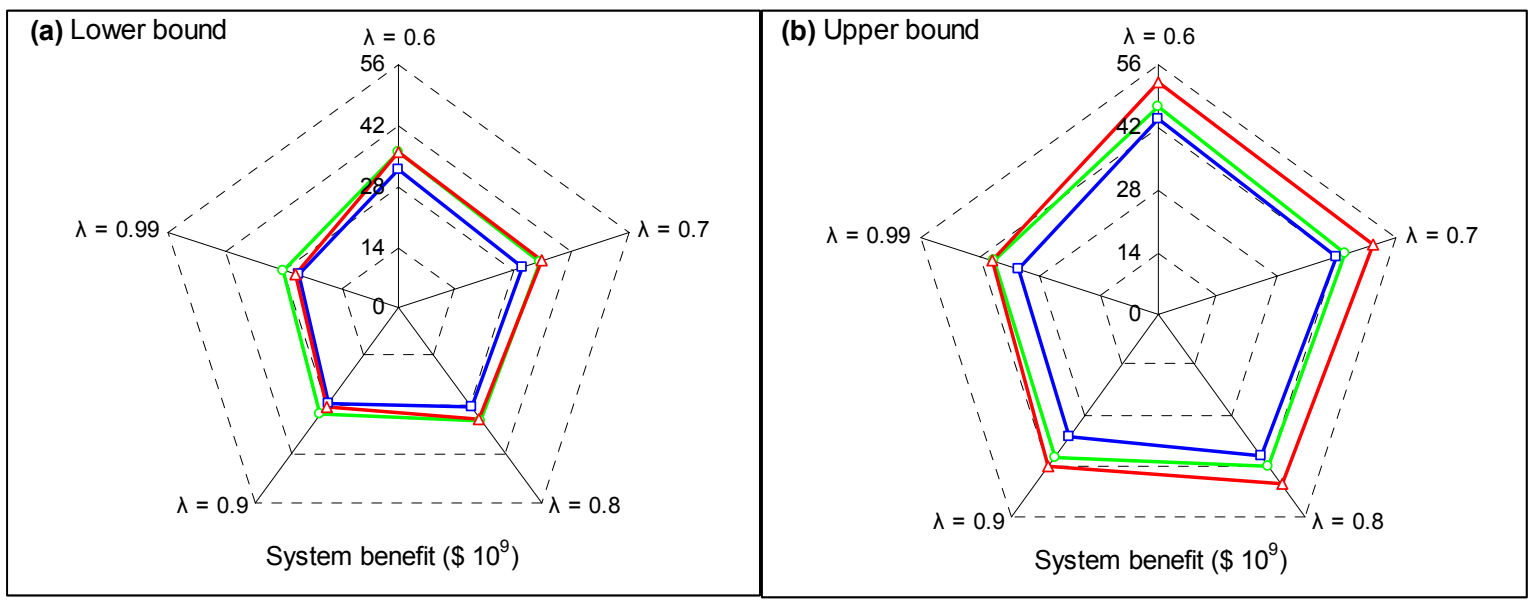

Figure 7. System benefits for scenarios 1-3.

\subsubsection{Strategies Analysis of Wet Land Expansion}

Since wet land plays a significant role in the regional sustainable development, which is not only suitable for diverting flooding to reduce losses, but also alleviate contradictions between water ecological 
preservation and economic development in water resources utilization. Policy analysis associated with different water resources management based on wet land expansion is essential to in-depth analyze, which would be used for identifying optimized water-allocation alternatives, lessening the losses of water deficits/floods, and achieving socioeconomic/ecological sustainability in study region. Therefore, another two scenarios based on actual water demand and land utilization structure in Dahuangbaowa floodplain (expressed in scenario 1) are considered. Scenario 2 assumed that wetland are expand 20\% (i.e., ecology water demand would increase), while the area of fishery and wet farm (in Zone 1 and 2) would reduce to remit the wetland; scenario 3 assumed that wetland are expanded by $40 \%$, while the area of dry farm, fruit farm, livestock and resident would reduce correspondent area to remit the wetland. Under these situations, different policies corresponding to varied shortages, surplus, targets, and allocations can be obtained, which can not only support water managers making a rational plan, but also improve the efficiencies of water resources allocations and damages reductions with consideration of water-use/land-use pattern, ecological protection and socioeconomic development.

Figure 8 presents water shortages and surplus under the three scenarios 1,2 and $3(\lambda=0.6)$, which indicated that water shortages and surplus would change by increasing of wetland expansion. In comparison, the shortages would decrease by wetland expansion, which indicates that losses of water deficiency would relieve by planning strategies. For example, the shortages would be $[76.2,165.2] \times 10^{6} \mathrm{~m}^{3}$ under scenario 1 , and $[0,154.8] \times 10^{6} \mathrm{~m}^{3}$ under scenario 3. Meanwhile, water surplus would increase due to wetland expansion, which results in reducing losses of system benefits since unit loss of surplus for ecology is lower than others (e.g., fishery, wet farm, dry farm, fruit farm, livestock and resident). For example, when water inflows is high level, the surplus would be [1897.6, 2294.8] $\times 10^{6} \mathrm{~m}^{3}$ under scenario 1 , and $2096.12 \times 10^{6} \mathrm{~m}^{3}$ under scenario 2 .
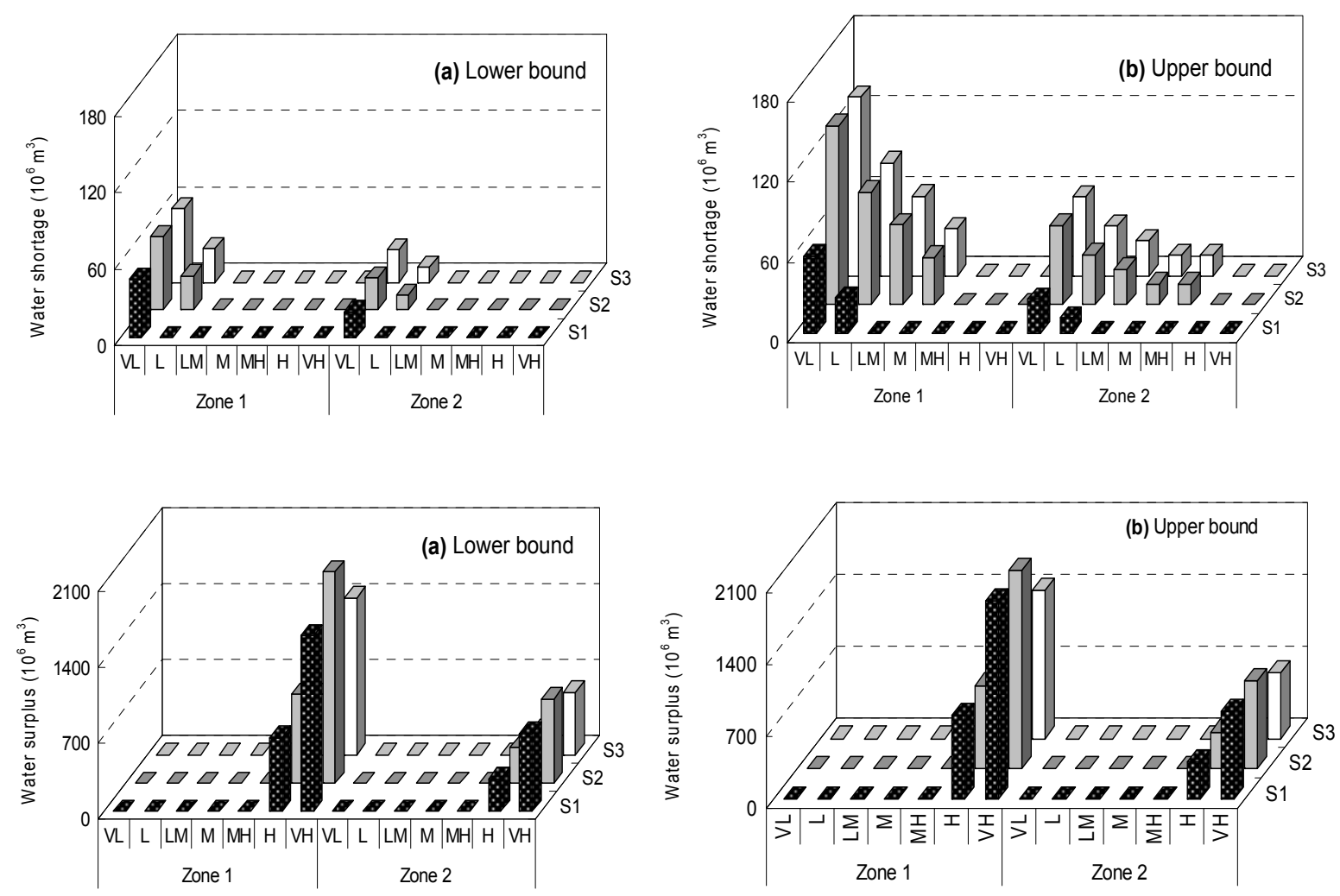

Figure 8. Water shortages and surplus of ecology under scenarios 1 to $3(\lambda=0.6)$. 
Meanwhile, since each allocated water flow is the difference between the promised target and the probabilistic shortage under a given stream condition with an associated probability level, which indicates that different wetland expansion would result in varied water-allocation patterns. Figure 9 presents the results for water allocations under the three scenarios $(\lambda=0.6)$, which indicates that allocated water would change by increasing of wetland expansion in floodplain. For example, in Zone 1, the water allocation would be $[2007.1,2066.5] \times 10^{6} \mathrm{~m}^{3}$ under scenario 1 , when water flow is very low level, while it would be $[2031.1,2072.3] \times 10^{6} \mathrm{~m}^{3}$ and $[2275.6,2697.9] \times 10^{6} \mathrm{~m}^{3}$ under scenarios 2 and 3. It implies that various strategies would result in variant water allocation. The results from Figures 8 and 9 imply that adjustment of planning strategies would increase the proportion of ecological water use, which results in reducing of losses (e.g., shortages and surplus losses) in system benefits.

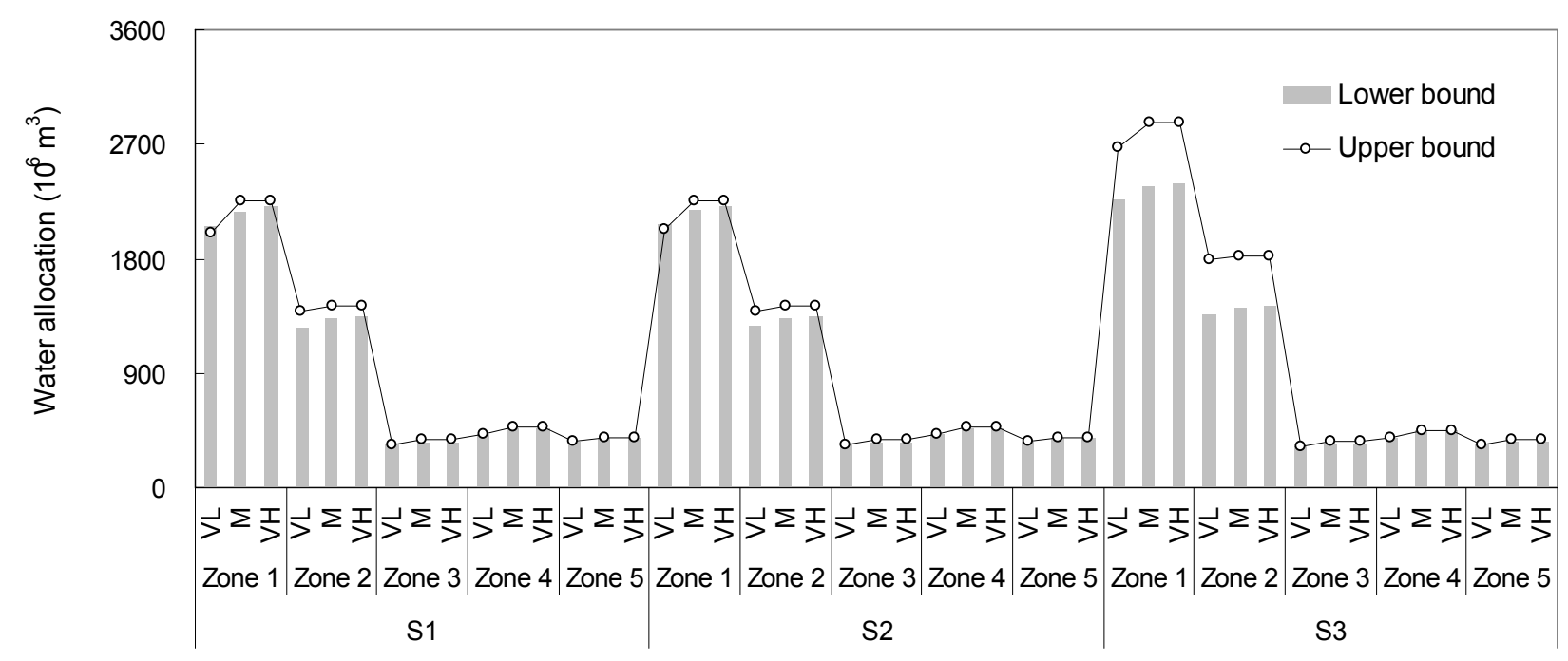

Figure 9. Water allocations under scenarios 1 to $3(\lambda=0.6)$.

Figure 7 presents system benefits under various scenarios, which reflect impacts of different policies (wetland expansion) on a water allocation system with considerations of flooding/non-flooding seasons. The lowest system benefits would be achieved under scenario $2\left([32.0,44.0] \times 10^{9} \$(\lambda=0.6)\right.$ and $\left.[27.9,34.0] \times 10^{9} \$(\lambda=0.9)\right)$, which indicates that the actual type of water demand and land utilization pattern (i.e., scenario 1) would be rational in floodplain; adjustment of ecology and other water consumers would result in reduction of system benefits. However, the adjustment of ecology and water consumers of flood buffer would achieve higher system benefits. For example, the system benefits would be $[36.0,52.1] \times 10^{9} \$(\lambda=0.6)$ and $[28.8,34.5] \times 10^{9} \$(\lambda=0.9)$ under scenario 2 . It implied that the expansion of wetland of the floodplain remitted by water demand of flood buffer would achieve a higher system benefit due to reduction of flood losses. In comparison, scenario 3 achieved highest system benefits among three scenarios, which indicates that expanding wetland in floodplain and reducing other user's water demand (e.g., dry farm, fruit farm, livestock and resident) in flood buffer can generate higher system benefits. However, in scenario 2, expanding wetland in floodplain and reducing user's water demand in floodplain (e.g., fishery and wet farm) is an inefficient manner, which generate lower system benefits than basic scenario (i.e., scenario 1). 


\subsection{Discussion}

In this study, IQT-WMMF has been developed for planning water resources in situations of limited data availability, of which the advantages can be summarized as follows: (i) it can deal with water resources planning problems resulting from randomness in water flow that expressed as a probabilistic manner due to data deficiencies; (ii) it also can reflect the tradeoffs between the predefined economic targets and the associated water shortage penalties/flood losses, as well as the fuzziness of the water availability (i.e., fuzzy manner); (iii) limited economic data can be expressed as interval numbers, which would be acceptable as its uncertain inputs; (iv) it can handle nonlinearity in cost/benefit objectives, and has a global optimum under a number of system conditions.

The applications of IQT-WMMF for water resources management and planning in Dahuangbaowa floodplain can support sustainable water resources management of floodplain, which are worth being taken-outs as follows: (a) the demarcation of floodplain places and flood buffer areas would result in various water-allocation alternatives, lessening the losses of water deficits and floods in such floodplain. Thus, the adjustment of land use is beneficial to identify optimized water-allocation alternatives, lessening the losses of water deficits/floods, and achieving socioeconomic and ecological development; (b) wet land plays a significant role in floodplain for diverting flooding to reduce losses, meanwhile it also can alleviate contradictions between water demands of human activities and ecological preservation. Therefore, policy analysis associated with wet land expansion can be advocated to support planning water resources sustainably; (c) limited data availability, error in statistics, cognitive limitation of human would influence efficiencies of water resources management, which would bring about enormous risks and challenges for water managers. The developed IQT-WMMF has proposed to help water managers to deal with uncertainties expressed as probability/profitability distribution in water flows simultaneously, and also handling nonlinearity and interval features in the water resources management. From above, an appropriate strategy effort for water resources planning and management can reduce the adverse effects of excessive human activities and flood disaster damage in a floodplain, particular in developing country or economy backward regions.

\section{Conclusions}

In this study, a mix inexact-quadratic two-stage water resources management of floodplain (IQT-WMMF) has been developed for regional sustainable development under uncertainty and limited data availability, through incorporating techniques of credibility-constrained programming (CP), two-stage programming (TP), interval-parameter programming (IPP) and quadratic programming (QP) within a general framework. The developed IQT-WMMF can deal with multiple uncertainties expressed as probability distributions and fuzzy sets in water resources allocation problems resulting from randomness in water availabilities; it also can promote capacities of fuzzy determination to offer a compromised decision; meanwhile it can resolve imprecise and no-linear economic data in WMF.

The developed method has been applied to a real case of planning water resources management of Dahuangbaowa floodplain, China. Water flows of the study region would be allocated by water manager to multiple users to satisfy water demand in no-flood seasons, while flood flows would be relived to submerge flood diversions when flood occur. Results indicate that the current water allocation pattern 
would result in higher water shortages in the flood buffer (i.e., zone 3 to 5) in a no-flooding period, while also resulting in relatively lower shortages in flood diversion (i.e., zone 1 to 2). Meanwhile, in flooding period, about $85 \%$ flood water would be adopted by flood diversion, thus, the damages of flood in flood buffer would be relative low. Since the acreage of ecology in flood diversion place (i.e., zone 1 to 2) reaches $68.2 \%$, the role of ecology for mitigating flood water is conspicuous. The results also indicate that net system benefit of ecological sector is lower than other water users, nevertheless, the losses of shortages and surplus are relative lower, which result in its good capacity and most economy when flood occurs. Under these situations, a number of strategies based on ecological expansions (wetland expansions) are assumed to compare, which discovers that scenario 3 would achieved the highest system benefit. Moreover, a number of scenarios based on different satisfaction degrees are listed to compare, which can help generating desired policies for water resources management with maximized economic benefits and minimized system-failure risks. In comparison, a lower credibility measure on constraint (i.e., $\alpha=0.6$ ) leads to a lower water shortage and a higher water allocation, which can reduce shortage of $12.25 \times 10^{6} \mathrm{~m}^{3}$ and increase allocation of $137.68 \times 10^{6} \mathrm{~m}^{3}$ than those under a higher one $(\alpha=0.9)$. The results indicate that a lower credibility measure expressed as a lower satisfaction degree and a higher system risk can generate higher in water allocation; while it would lead to a higher risk in such a water resources management system. Therefore, the local decision makers need to adjust water-allocation policy based on the regional situation (e.g., climate change, population growth, economy development, and water demand increase) to balance the tradeoff between the system benefit and water shortage risk.

Although the developed IQT-WMMF model can effectively deal with uncertainties existing in the planning problems for regional sustainable development, there are also several limitations of the proposed method that are subject to further improvement. For example, in the practical water resources management problems, multiple uncertainties (e.g., random in water availability, varied water demand, imprecise economic data, different allocation target) generate complex uncertainties and various risks in water resources system, which affect efficiency of water policies of satisfactions and optimistic/pessimistic criterion for water resources planning. Therefore, IQT-WMMF would be integrated with robust optimization techniques to enhance its capacities in tackling uncertainties presented in multiple formats.

\section{Acknowledgments}

This research was supported by the Natural Sciences Foundation of China (Grant No. 41171405). The authors are grateful to the editors and the anonymous reviewers for their insightful comments and suggestions.

\section{Author Contributions}

Main text paragraph is written by Xueting Zeng and Xiaoliu Yang. Yuli Yang is in charge of polishing English presentation. Huili Chen is responsible for preparing the basic data and GIS graphics.

\section{Conflicts of Interest}

We declare that we do not have any commercial or associative interest that represents a conflict of interest in connection with the work submitted. 


\section{Appendix}

$$
\begin{aligned}
\operatorname{Max} f^{+}= & {\left[\sum_{j=1}^{J_{1}} a_{j}^{+}\left(x_{j}^{+}\right)^{2}+\sum_{j=q_{1}+1}^{n_{1}} a_{j}^{+}\left(x_{j}^{-}\right)^{2}+\sum_{j=1}^{J_{1}} b_{j}^{+} x_{j}^{+}+\sum_{j=q_{1}+1}^{n_{1}} b_{j}^{+} x_{j}^{-}\right] } \\
& -\left\{\sum_{h=1}^{H} p_{t h}\left[\sum_{j=1}^{J_{2}} c_{t h}^{-}\left(y_{j t h}^{-}\right)^{2}-\sum_{j=q_{2}+1}^{n_{2}} c_{t h}^{-}\left(y_{j t h}^{+}\right)^{2}-\sum_{j=1}^{J_{2}} e_{t h}^{-} y_{j t h}^{-}-\sum_{j=q_{2}+1}^{n_{2}} e_{t h}^{-} y_{j t h}^{+}\right]\right\}
\end{aligned}
$$

subject to

$$
\begin{gathered}
\sum_{j=1}^{q_{1}}\left|A_{i j}\right|^{-} \operatorname{sign}\left(A_{i j}^{-}\right) x_{i j}^{+}+\sum_{j=q_{1}+1}^{n_{1}}\left|A_{i j}\right|^{+} \operatorname{sign}\left(A_{i j}^{+}\right) x_{i j}^{-} \leq B_{i}+\left(1-2 \lambda_{i}\right)\left(B_{i}-\underline{B_{i}}\right), \\
\forall \mathrm{i}=1,2, \ldots, \mathrm{M}, \quad i=1,2, \ldots, m_{1} \\
\sum_{j=1}^{J_{1}}\left|A_{r j}\right|^{-} \operatorname{sign}\left(A_{r j}^{-}\right) x_{j}^{+}+\sum_{j=q_{1}+1}^{n_{1}}\left|A_{r j}\right|^{+} \operatorname{sign}\left(A_{r j}^{+}\right) x_{j}^{-}+\sum_{j=1}^{J_{2}}\left|A_{r j}^{\prime}\right|^{-} \operatorname{sign}\left(A_{j}^{++}\right) y_{j h}^{-} \\
+\sum_{j=q_{1}+1}^{n_{2}}\left|A_{r j}^{\prime}\right|^{+} \operatorname{sign}\left(A_{r j}^{-}\right) y_{j h}^{-} \leq w_{j h}^{+}, \quad r=1,2, \ldots, m_{2} ; \quad h=1,2, \ldots, H \\
x_{j t}^{+} \geq 0, \quad j=1,2, \ldots, J_{1} \\
x_{j t}^{-} \geq 0, \quad j=J_{1}+1, \ldots, n_{1} \\
y_{j t h}^{+} \geq 0, \quad j=1,2, \ldots, J_{2} \\
y_{j t h}^{-} \geq 0, \quad j=J_{2}+1, \ldots, n_{2}, \quad h=1,2, \ldots, H \\
\operatorname{Max} f^{-}=\left[\sum_{j=1}^{J_{1}} a_{j}^{-}\left(x_{j}^{-}\right)^{2}+\sum_{j=q_{1}+1}^{n_{1}} a_{j}^{-}\left(x_{j}^{+}\right)^{2}+\sum_{j=1}^{J_{1}} b_{j}^{-} x_{j}^{-}+\sum_{j=q_{1}+1}^{n_{1}} b_{j}^{-} x_{j}^{+}\right] \\
-\left\{\sum_{h=1}^{H} p_{t h}\left[\sum_{j=1}^{J_{2}} c_{t h}^{+}\left(y_{j t h}^{+}\right)^{2}-\sum_{j=q_{2}+1}^{n_{2}} c_{t h}^{+}\left(y_{j t h}^{-}\right)^{2}-\sum_{j=1}^{J_{2}} e_{t h}^{+} y_{j t h}^{+}-\sum_{j=q_{2}+1}^{n_{2}} e_{t h}^{+} y_{j t h}^{-}\right]\right\}
\end{gathered}
$$

subject to

$$
\begin{gathered}
\sum_{j=1}^{q_{1}}\left|A_{i j}\right|^{+} \operatorname{sign}\left(A_{i j}^{+}\right) x_{i j}^{-}+\sum_{j=q_{1}+1}^{n_{1}}\left|A_{i j}\right|^{-} \operatorname{sign}\left(A_{i j}^{-}\right) x_{i j}^{+} \leq B_{i}+\left(1-2 \lambda_{i}^{-}\right)\left(B_{i}-\underline{B_{i}}\right), \\
\forall \mathrm{i}=1,2, \ldots, \mathrm{M}, \quad i=1,2, \ldots, m_{1} \\
\sum_{j=1}^{J_{1}}\left|A_{r j}\right|^{+} \operatorname{sign}\left(A_{r j}^{+}\right) x_{j}^{-}+\sum_{j=q_{1}+1}^{n_{1}}\left|A_{r j}\right|^{-} \operatorname{sign}\left(A_{r j}^{-}\right) x_{j}^{+}+\sum_{j=1}^{J_{2}}\left|A_{r j}^{\prime}\right|^{+} \operatorname{sign}\left(A_{j}^{\prime-}\right) y_{j h}^{+} \\
+\sum_{j=q_{1}+1}^{n_{2}}\left|A_{r j}^{\prime}\right|^{-} \operatorname{sign}\left(A_{r j}^{++}\right) y_{j h}^{+} \leq w_{j h}^{-}, \quad r=1,2, \ldots, m_{2} ; \quad h=1,2, \ldots, H \\
0 \leq x_{j t}^{-} \leq x_{j t o p t}^{+}, \quad j=1,2, \ldots, J_{1} \\
x_{j t}^{+} \geq x_{j t o p t}^{-}, \quad j=J_{1}+1, \ldots, n_{1} \\
0 \leq y_{j t h}^{-} \leq y_{j t h o p t}^{+}, \quad j=J_{1}+1, \ldots, n_{2}
\end{gathered}
$$




$$
\begin{gathered}
y_{j t h}^{+} \geq y_{j t h o p t}^{-}, \quad j=1,2, \ldots, J_{2} \\
y_{j t h}^{+} \geq 0, \quad j=1,2, \ldots, J_{2} \\
y_{j t h}^{-} \geq 0, \quad j=J_{2}+1, \ldots, n_{2}
\end{gathered}
$$

Thereby, optimal solutions of Model (7) can be gained, including $x_{j t o p t}^{-}$for $j=1$ to $J_{1}, x_{j t o p t}^{+}$for $j=J_{1}+1$ to $n_{1}, y_{\text {jthopt }}^{-}$for $k=1$ to $J_{2}$, and $y_{\text {jthopt }}^{+}$for $k=J_{2}+1$ to $\mathrm{n}_{2}$. Through integrating the solutions of the two submodels, the solution of IQT model can be generated [15].

\section{References}

1. Guo, P.; Huang, G.H.; Li, Y.P. An inexact fuzzy-chance-constrained two-stage mixed-integer linear programming approach for flood diversion planning under multiple uncertainties. Adv. Water Resour. 2010, 33, 81-89.

2. Maqsood, I. Development of Simulation- and Optimization-Based Decision Support Methodologies for Environmental Systems Management. Ph.D. Thesis, University of Regina, Regina, SK, Canada, 2004.

3. Wang, X.H.; Du, C.M. An internet based flood warning system. J. Environ. Inform. 2003, 2, 48-56.

4. Sun, L.Y.; Christensen, P.; Liu, J.L. Comparative study of water resource management and policies for ecosystems in China and Denmark. J. Environ. Inform. 2013, 21, 72-83.

5. Li, Y.P.; Huang, G.H.; Nie, S.L. A robust interval-based minimax-regret analysis approach for the identification of optimal water-resources-allocation strategies under uncertainty. Resour. Conserv. Recycl. 2009, 54, 86-96.

6. Li, Y.P.; Huang, G.H.; Yang, Z.F.; Nie, S.L. Interval-Fuzzy multistage programming for water resources management under uncertainty. Resour. Conserv. Recycl. 2008, 52, 800-812.

7. Li, Y.P.; Huang, G.H.; Chen, X. Multistage scenario-based interval-stochastic programming for planning water resources allocation. Stoch. Environ. Res. Risk Assess. 2009, 23, 781-792.

8. Correia, F.N.; Rego, F.; Saraiva, M.G.; Bernardo, F. Flood hazard assessment and management: Interface with the public. Water Resour. Manag. 1998, 12, 209-217.

9. Olsen, J.R.; Beling, P.A.; Lambert, J.H. Dynamic models for floodplain management. J. Water Resour. Plan. Manag. 2000, 126, 167-171.

10. Seifi, A.; Hipel, K.W. Interior-Point method for reservoir operation with stochastic inflows. J. Water Resour. Plan. Manag. 2001, 127, 48-57.

11. Nazemi, A.R.; Akbarzadeh, M.R.; Hosseini, S.M. Fuzzy-Stochastic linear programming in water resources engineering. Stoch. Environ. Res. Risk Assess. 2002, 2, 167-174.

12. Ni, J.R.; Xue, A. Application of artificial neural network to the rapid feed-back of potential ecological risk in flood diversion zone. Eng. Appl. Artif. Intell. 2003, 16, 105-119.

13. Douben, K.J. Characteristics of river floods and flooding: A global overview, 1985-2003. Irrig. Drain. 2006, 55, 9-21.

14. Jiménez, M.; Arenas, M.; Bilbao, A. Linear programming with fuzzy parameters: An interactive method resolution. Eur. J. Oper. Res. 2007, 177, 1599-1609. 
15. Fan, Y.R.; Huang, G.H.; Yang, A.L. Generalized fuzzy linear programming for decision making under uncertainty: Feasibility of fuzzy solutions and solving approach. Inform. Sci. 2013, 241, 12-27.

16. Maqsood, I.; Huang, G.H.; Huang, Y. ITOM: An interval-parameter two-stage optimization model for stochastic planning of water resources systems. Stoch. Environ. Res. Risk Assess. 2005, 19, $125-133$.

17. Kenneth, W.H. Two-Stage decision-making under uncertainty and stochasticity: Bayesian Programming. Adv. Water Resour. 2007, 30, 641-664.

18. Gómez, C.M.; Pérez, B.C.D. Do drought management plans reduce drought risk? A risk assessment model for a Mediterranean river basin. Ecol. Econ. 2012, 76, 42-48.

19. Freeze, R.A.; Massmann, J.; Smith, L.; Sperling, T.; James, B. Hydrogeological decision analysis: 1. A framework. Ground Water 1990, 28, 738-766.

20. Li, Y.P.; Huang, G.H. Fuzzy-Stochastic-Based violation analysis method for planning water resources management systems with uncertain information. Inform. Sci. 2009, 179, 4261-4276.

21. Zeng, X.; Li, Y.; Huang, G.; Yu, L. Inexact mathematical modeling for the identification of water trading policy under uncertainty. Water 2014, 6, 229-252.

22. Zhang, Y.M.; Huang, G.H. Optimal water resource planning under fixed budget by interval-parameter credibility constrained programming. Eng. Optim. 2011, 43, 879-889.

23. Hillier, F.S.; Lieberman, G.J. Introduction to Operations Research, 4th ed.; Holden-Day: Oakland, CA, USA, 1986.

24. Li, Y.P.; Huang, G.H.; Nie, S.L.; Mo, D.W. Interval-Parameter robust quadratic programming for water quality management under uncertainty. Eng. Optim. 2008, 40, 613-635.

25. Chen, M.J.; Huang, G.H. A derivative algorithm for inexact quadratic program-application to environmental decision-making under uncertainty. Eur. J. Oper. Res. 2001, 128, 570-586.

26. Li, Y.P.; Huang, G.H.; Xiao, H.N.; Qin, X.S. An inexact two-stage quadratic program for water resources planning. J. Environ. Inform. 2007, 10, 99-105.

27. Vylder, F.D.; Sundt, B. Constrained credibility estimators in the regression model. Scand. Actuar. J. 1982, 1, 27-31.

28. Huang, X. Credibility-Based chance-constrained integer programming models for capital budgeting with fuzzy parameters. Inform. Sci. 2006, 176, 2698-2712.

29. Liu, B.; Liu, Y.K. Expected value of fuzzy variable and fuzzy expected value models. IEEE Trans. Fuzzy Syst. 2002, 10, 45-50.

30. Zeng, X.T.; Li, Y.P.; Huang, W.; Bao, A.M.; Chen, X. Two-Stage credibility-constrained programming with Hurwicz criterion (TCP-CH) for planning water resources management. Eng. Appl. Artif. Intell. 2014, 35, 164-175.

31. Trumbo, C.W.; McComa, K.A. The Function of Credibility in Information Processing for Risk Perception. Risk Anal. 2003, 23, 343-353.

32. Punyangarm, V.; Yanpirat, P.; Charnsethikul, P.; Lertworasirikul, S. A Credibility Approach for Fuzzy Stochastic Data Envelopment Analysis (FSDEA). In Proceedings of the 7th Asia Pacific Industrial Engineering and Management Systems Conference, Bangkok, Thailand, 17-20 December 2006.

33. Zhang, Y.M.; Huang, G.H. Inexact credibility constrained programming for environmental system management. Resour. Conserv. Recycl. 2011, 55, 1-7. 
34. Li, Z.; Huang, G.; Zhang, Y.; Li, Y. Inexact two-stage stochastic credibility constrained programming for water quality management. Resour. Conserv. Recycl. 2013, 73, 122-132.

35. Fan, Y.R.; Huang, G.H. A robust two-step method for solving interval linear programming problems within an environmental management context. J. Environ. Inform. 2012, 19, 1-9.

36. The Water Resources Bulletin of Tianjin 2000; Tianjing Water Conservancy Bureau: Tianjing, China, 2001.

37. The Water Resources Bulletin of Tianjin 2007; Tianjing Water Conservancy Bureau: Tianjing, China, 2008.

38. The Water Resources Bulletin of Tianjin 2011; Tianjing Water Conservancy Bureau: Tianjing, China, 2012.

39. The Water Resources Bulletin of Tianjin 2013; Tianjing Water Conservancy Bureau: Tianjing, China, 2014.

40. Huang, Y.; Li, Y.P.; Chen X.; Bao, A.M.; Zhou, M. Simulation-based optimization method for water resources management in Tarim River Basin, China. Sci. Director 2010, 2, 1451-1460.

41. The Statistical Yearbook of Tianjin 2000; Tianjing Bureau of statistics: Tianjing, China, 2001.

42. The Statistical Yearbook of Tianjin 2007; Tianjing Bureau of statistics: Tianjing, China, 2008.

43. The Statistical Yearbook of Tianjin 2011; Tianjing Bureau of statistics: Tianjing, China, 2012.

44. The Statistical Yearbook of Tianjin 2013; Tianjing Bureau of statistics: Tianjing, China, 2014.

45. The Second National Land Survey; Ministry of land resources: Beijing, China, 2009.

(C) 2015 by the authors; licensee MDPI, Basel, Switzerland. This article is an open access article distributed under the terms and conditions of the Creative Commons Attribution license (http://creativecommons.org/licenses/by/4.0/). 